JOURNAL OF THE

AMERICAN MATHEMATICAL SOCIETY

Volume 13, Number 1, Pages 55-82

S 0894-0347(99)00311-2

Article electronically published on July 20, 1999

\title{
COMPUTING ROADMAPS OF SEMI-ALGEBRAIC SETS ON A VARIETY
}

\author{
SAUGATA BASU, RICHARD POLLACK, AND MARIE-FRANCOISE ROY
}

\section{INTRODUCTION}

Let $\mathbf{R}$ be a real closed field, $Z(Q)$ a real algebraic variety defined as the zero set of a polynomial $Q \in \mathbf{R}\left[X_{1}, \ldots, X_{k}\right]$ and $S$ a semi-algebraic subset of $Z(Q)$, defined by a Boolean formula with atoms of the form $P<0, P>0, P=0$ with $P \in \mathcal{P}$, where $\mathcal{P}$ is a finite subset of $\mathbf{R}\left[X_{1}, \ldots, X_{k}\right]$.

A semi-algebraic set $C$ is semi-algebraically connected if it is non-empty and is not the union of two non-empty disjoint semi-algebraic sets which are closed and open in $C$. A semi-algebraically connected component of $S$ is a semi-algebraic subset of $S$ which is semi-algebraically connected, and closed and open in $S$. Semi-algebraic sets have a finite number of semi-algebraically connected components ([5], page 34). A roadmap of $S$, which we denote $R(S)$, is a semi-algebraic set of dimension at most one contained in $S$ which satisfies the roadmap conditions:

RM1 For every semi-algebraically connected component $C$ of $S, C \cap R(S)$ is semialgebraically connected.

RM2 For every $x \in \mathbf{R}$, and for every semi-algebraically connected component $C^{\prime}$ of $S_{x}, C^{\prime} \cap R(S) \neq \emptyset$.

Here, and everywhere else in this paper, $\pi$ is the projection on the first coordinate and for $X \subset \mathbf{R}, S_{X}$ is $S \cap \pi^{-1}(X)$. We also use the abbreviations $S_{x}, S_{<c}$, and $S_{\leq c}$ for $S_{\{x\}}, S_{(-\infty, c)}$, and $S_{(-\infty, c]}$ respectively. Algorithms for the construction of roadmaps are described in terms of the parameters $k, k^{\prime}, s, d$ where $k$ is the dimension of the ambient space, $k^{\prime}$ is the dimension of $Z(Q), s$ is the number of polynomials in $\mathcal{P}$ and $d$ is a bound on the degrees of the polynomials in $\mathcal{P}$ and the polynomial $Q$.

Given a roadmap $R(S)$ and a point $p \in S$ the connecting subroutine outputs a semi-algebraic continuous path in $S_{\pi(p)}$ connecting $p$ to $R(S)$. The connecting subroutine is described in terms of the parameters $k, k^{\prime}, s, d$ as before and $\tau$ which is a bound on the degrees of the polynomials defining $p$ (see section 4 for a discussion of how points are described by polynomials).

Received by the editors November 25, 1997 and, in revised form, April 14, 1999.

1991 Mathematics Subject Classification. Primary 14P10,68Q25; Secondary 68Q40.

Key words and phrases. Roadmaps, semi-algebraic sets, variety.

The first author was supported in part by NSF Grants CCR-9402640 and CCR-9424398.

The second author was supported in part by NSF Grants CCR-9402640, CCR-9424398, DMS9400293, CCR-9711240 and CCR-9732101.

The third author was supported in part by the project ESPRIT-LTR 21024 FRISCO and by European Community contract CHRX-CT94-0506. 
The roadmap is stored as a graph whose vertices are points of $S$ and whose edges are semi-algebraic curves. Thus, the roadmap and the connecting subroutine enable us to decide whether or not two points $x$ and $y$ of $S$ are in the same semialgebraically connected component of $S$. Furthermore, if $x$ and $y$ are in the same semi-algebraically connected component, we obtain a semi-algebraic path joining the two points which consists of certain branches of the roadmap and of the connecting paths.

The problem of deciding connectivity properties of semi-algebraic sets has attracted much attention. One motivation comes from robot motion planning (see [23]) where the free space of a robot (the subspace of the configuration space of the robot consisting of those configurations where the robot is neither in conflict with its environment nor itself) can be modeled as a semi-algebraic set. In this context it is important to know whether a robot can move from one configuration to another. This is equivalent to deciding whether the two corresponding points in the free space are in the same connected component of the free space. This problem was solved by Schwartz and Sharir [23] using Collins' method of cylindrical algebraic decomposition [9]. The complexity of their solution is polynomial in $d$ and $s$ and doubly exponential in $k$. In 1987, John Canny [6] introduced the notion of a roadmap of a semi-algebraic set. He gave an algorithm $[6,7]$ constructing a roadmap for a semi-algebraic set whose complexity is $s^{k}(\log s) d^{O\left(k^{4}\right)}$. Since the given semi-algebraic set might have $(s d)^{k}$ different connected components, the part of the complexity depending on $s$ in Canny's algorithm is nearly optimal. Further developments can be found in $[12,15,8,16,13,2]$.

In robot motion planning, the configuration space of a robot is often a semialgebraic subset confined to be a subset of an algebraic variety whose dimension is significantly smaller than the dimension of the real Euclidean space in which it is naturally embedded (see for example [17], page 65). In such a situation it is of interest to design algorithms which take advantage of this fact and whose complexity reflects the dimension of this variety rather than the dimension of the ambient space. It is for this reason that we consider the problem of constructing a roadmap on a semi-algebraic subset of a variety $Z(Q)$. We will present several algorithms and whenever we mention their complexity it is the number of arithmetic operations and comparisons in the ring generated by the coefficients of the input polynomials. We present an algorithm for computing a roadmap of a semi-algebraic set $S \subset Z(Q)$ defined by polynomials in $\mathcal{P}$ with complexity $s^{k^{\prime}+1} d^{O\left(k^{2}\right)}$. Note that the complexity of the algorithm is separated into a combinatorial part depending on $s$ and $k^{\prime}$ and an algebraic part depending on $d$ and $k$. We also present an algorithm which given a point of $S$ defined by a polynomial of degree at most $\tau$ constructs a path joining this point to the roadmap. The complexity of this algorithm is $k^{\prime} s \tau^{O(1)} d^{O\left(k^{2}\right)}$. In the case of input polynomials with integer coefficients of size bounded by $b$ the bit complexity for computing the roadmap is $b^{*} s^{k^{\prime}+1} d^{O\left(k^{2}\right)}$ and the bit complexity for connecting a point is $b^{*} k^{\prime} s \tau^{O(1)} d^{O\left(k^{2}\right)}$, where $b^{*}=b \log b \log \log b$. A preliminary version of the present paper appeared in [4].

The rest of the paper is organized as follows. In section 2 we introduce the notions of pseudo-critical values and special values, and present some results about them that are crucial for our algorithms. In section 3 we give precise descriptions of the input, output and complexity of several subroutines that we use in our algorithm. Next, in section 4, we develop some algorithmic tools that enable us 
to compute curves in a given algebraic set parametrized by the first coordinates and to identify certain distinguished slices where our algorithm calls itself in one lower dimension. In section 5 we present an algorithm which given an algebraic set $Z(Q)$ and a finite subset $\mathcal{M}$ of $Z(Q)$ constructs a roadmap of $Z(Q)$ which contains $\mathcal{M}$. In section 6 we introduce the notion of combinatorial level. It controls the complexity of an algorithm that constructs a uniform roadmap. In section 7 we present a roadmap algorithm for an arbitrary semi-algebraic set $S$ defined by the set of polynomials $\mathcal{P}$ and lying in a bounded algebraic set $Z(Q)$. The idea is to construct uniform roadmaps for a perturbed finite set of polynomials on various approximating varieties close to $Z(Q)$, for which the combinatorial level is $k^{\prime}$ and to take the limit of the curves so constructed when the parameters of perturbation tend to 0 . In order to ensure that the curve so constructed is connected in every connected component, we need to add curves connecting well chosen points to the various roadmaps on the approximating varieties. In section 8 , we solve the same problem without the assumption that the variety $Z(Q)$ is bounded.

In order to assist the reader in section 3 , we primarily cite the paper [1] for its ease of accessibility.

\section{Preliminaries}

If $\mathcal{P}$ is a finite subset of $\mathbf{R}\left[X_{1}, \ldots, X_{k}\right]$, then we write $Z(\mathcal{P})$ for the set of common zeroes in $\mathbf{R}^{k}$ of the polynomials in $\mathcal{P}$. Note that, in a real closed field any algebraic set can be defined by a single equation by taking sums of squares. Thus, $Z(\mathcal{P})=Z\left(\sum_{Q \in \mathcal{P}} Q^{2}\right)$. We use $B(x, r)$ and $S(x, r)$ to denote the open ball and sphere of center $x$ and radius $r$ respectively. Let $Z(Q) \subset \mathbf{R}^{k}$ be a smooth algebraic hypersurface, i.e. an algebraic set defined by a polynomial $Q$ such that

$$
\operatorname{Grad}(Q)=\left(\frac{\partial Q}{\partial X_{1}}, \ldots, \frac{\partial Q}{\partial X_{k}}\right)
$$

is never 0 on $Z(Q)$. A critical point of $\pi$ on $Z(Q)$ is a point of $Z(Q)$ at which the gradient of $Q$ is parallel to the $X_{1}$-axis, and a critical value of $\pi$ on $Z(Q)$ is the projection on the $X_{1}$-axis of a critical point of $\pi$ on $Z(Q)$. An immediate consequence of the semi-algebraic implicit function theorem over a real closed field ([5], page 56) is the following.

Proposition 1. If $x$ is not a critical point of $\pi$ on $Z(Q)$, then for $r$ small enough $Z(Q) \cap B(x, r)_{<\pi(x)}$ is semi-algebraically connected.

The following property, well known over the field of real numbers $\mathbb{R}$, is also true over any real closed field $\mathbf{R}$ [11].

Proposition 2. Let $Z(Q)$ be a smooth algebraic hypersurface and let $C$ be a bounded semi-algebraically connected component of $Z(Q)_{[a, b]}$ on which $\pi$ has no critical value in $[a, b]$. Then, for every $d \in[a, b], C_{d}$ is semi-algebraically connected.

Since the algebraic set $Z(Q)$ may not be a smooth hypersurface we shall perturb the polynomial $Q$ in such a way that the zero set of the perturbed polynomial is a smooth hypersurface and the connectivity properties of $Z(Q)$ will be controlled by pseudo-critical values which are the limits of critical values on the perturbed smooth hypersurface. More precisely, we will perturb polynomials by infinitesimals as follows. We write $\mathbf{R}\langle\zeta\rangle$ for the real closed field of algebraic Puiseux series in $\zeta$ with coefficients in $\mathbf{R}$ ([5], pages 11 and 16). The sign of such an algebraic Puiseux 
series agrees with the sign of the coefficient of its lowest degree term in $\zeta$. This order makes $\zeta$ positive and infinitesimal: its absolute value is smaller than any positive element of $\mathbf{R}$. The map $\lim _{\zeta}$ maps an element of $\mathbf{R}\langle\zeta\rangle$ bounded over $\mathbf{R}$, one that has no negative powers of $\zeta$, to its constant term. An element of $\mathbf{R}\langle\zeta\rangle$ is infinitesimal over $\mathbf{R}$ if it is mapped by $\lim _{\zeta}$ to 0 . If $S$ is a semi-algebraic subset of $\mathbf{R}^{k}$, we denote by $S_{\mathbf{R}\langle\zeta\rangle}$ the subset of $\mathbf{R}\langle\zeta\rangle^{k}$ defined by the same equalities and inequalities that define $S$. An immediate consequence of the fact that a semi-algebraic subset of a real closed field is a finite union of points and intervals is the following:

Proposition 3. Let $\Phi$ be a first order sentence of the language of ordered fields with coefficients in $\mathbf{R}[\zeta]$, and let $\Phi(t)$ be the sentence obtained by substituting $t \in \mathbf{R}$ for $\zeta$ in $\Phi$. Then $\Phi$ is true in $\mathbf{R}\langle\zeta\rangle$ if and only if there exists $t_{0}$ in $\mathbf{R}$ such that for every $t \in\left(0, t_{0}\right), \Phi(t)$ is true in $\mathbf{R}$.

Let $S$ be a semi-algebraic subset of $\mathbf{R}\langle\zeta\rangle^{k}$ defined by polynomials in $k$ variables with coefficients in $\mathbf{R}[\zeta]$ which is non-empty, closed, and bounded over $\mathbf{R}$. Let $S^{\prime}$ be the semi-algebraic subset of $\mathbf{R}^{k+1}$ defined by substituting a new first variable $T$ for $\zeta$ in the definition of $S$. The following is proved in [1] (see also [21]).

Proposition 4. The set $\lim _{\zeta}(S)$ is the intersection of the closure of $S_{>0}^{\prime}$ with the hyperplane $T=0$. If $S$ is semi-algebraically connected, then $\lim _{\zeta}(\bar{S})$ is semialgebraically connected.

Given a bounded algebraic set $Z(Q) \subset B(0, M) \subset \mathbf{R}^{k}$ defined by a polynomial $Q$ of degree $\leq d$, we adapt an idea of Gournay-Risler [13] and consider $Z\left(Q_{1}\right) \subset \mathbf{R}\langle\zeta\rangle^{k}$ and $Z\left(Q_{2}\right) \subset \mathbf{R}\langle\zeta\rangle^{k+1}$ where

$$
\begin{gathered}
Q_{1}=(1-\zeta) Q^{2}+\zeta\left(X_{1}^{2 d+2}+\cdots+X_{k}^{2 d+2}-k M^{2 d+2}\right), \\
Q_{2}=(1-\zeta)\left(Q^{2}+X_{k+1}^{2}\right)+\zeta\left(X_{1}^{2 d+2}+\cdots+X_{k}^{2 d+2}+X_{k+1}^{4}-k M^{2 d+2}\right) .
\end{gathered}
$$

The algebraic set $Z\left(Q_{1}\right)$ is a smooth algebraic hypersurface of $\mathbf{R}\langle\zeta\rangle^{k}$ bounded over $\mathbf{R}$ on which $\pi$ has a finite number of critical points [3].

Similarly, the algebraic set $Z\left(Q_{2}\right)$ is a smooth algebraic hypersurface of $\mathbf{R}\langle\zeta\rangle^{k+1}$ bounded over $\mathbf{R}$ on which $\pi$ has a finite number of critical points. Note that a critical point of $\pi$ on $Z\left(Q_{2}\right)$ must have its last coordinate 0 and thus its first $k$ coordinates define a critical point of $\pi$ on $Z\left(Q_{1}\right)$.

Lemma 1. Let $Z(Q) \subset B(0, M)$ be a bounded algebraic set. Then, $\lim _{\zeta}\left(Z\left(Q_{1}\right)\right)=$ $\lim _{\zeta}\left(Z\left(Q_{2}\right)\right)=Z(Q)$.

Proof. It is clear that $\lim _{\zeta}\left(Z\left(Q_{1}\right)\right) \subset Z(Q)$. To show that $Z(Q) \subset \lim _{\zeta}\left(Z\left(Q_{1}\right)\right)$ we make the following observations. Given $x \in Z(Q)$, for every $r>0$ in $\mathbf{R}$ there is a $y \in B(x, r)$ such that $Q(y) \neq 0$. From this we conclude that $Q_{1}(x)<0$ and $Q_{1}(y)>0$. Hence there exists $z \in \mathbf{R}\langle\zeta\rangle^{k}$ such that $Q_{1}(z)=0$ and $\lim _{\zeta}(z)=x$. The proof for $Z\left(Q_{2}\right)$ is entirely similar.

The algebraic set $Z\left(Q_{2}\right)$ has the following property which is not always enjoyed by $Z\left(Q_{1}\right)$.

Lemma 2. Let $Z(Q) \subset B(0, M)$ be a bounded algebraic set. For every semialgebraically connected component $C$ of $Z(Q)_{[a, b]}$ there exists a semi-algebraically connected component $D$ of $Z\left(Q_{2}\right)_{[a, b]}$ such that $\lim _{\zeta}(D)=C$. 
Proof. Let $x=\left(x_{1}, \ldots, x_{k}\right)$ be a point of $C_{\mathbf{R}\langle\zeta\rangle}$. There exists a unique point $f(x)=\left(x_{1}, \ldots, x_{k}, x_{k+1}\right)$ in $Z\left(Q_{2}\right)$ for which $x_{k+1}>0$. The mapping $f$ is semialgebraically continuous. Moreover if $x=\left(x_{1}, \ldots, x_{k}\right)$ is in $C, x_{k+1} \in \mathbf{R}\langle\zeta\rangle$ is infinitesimal over $\mathbf{R}$. Thus for every $y$ in $C$, the point $f(y)$ belongs to the connected component $D$ of $Z\left(Q_{2}\right)_{[a, b]}$ containing $f(x)$. Since $\lim _{\zeta}(D)$ is connected (Proposition 4) and contained in $Z(Q), \lim _{\zeta}(D)=C$.

A pseudo-critical point of $\pi$ on $Z(Q)$ is the $\lim _{\zeta}$ of a critical point of $\pi$ on $Z\left(Q_{1}\right)$. Note that a pseudo-critical point of $\pi$ on $Z(Q)$ is also the $\lim _{\zeta}$ of a critical point of $\pi$ on $Z\left(Q_{2}\right)$. A pseudo-critical value of $\pi$ on $Z(Q)$ is the projection on the $X_{1}$-axis of a pseudo-critical point of $\pi$ on $Z(Q)$. Note that the set of pseudo-critical values of $\pi$ on $Z(Q)$ is finite since the number of critical points of $\pi$ on $Z\left(Q_{1}\right)$ is finite.

Proposition 5. Let $Z(Q) \subset B(0, M)$ be a bounded algebraic set with $[a, b] \subset$ $[-M, M]$. Let $C$ be a semi-algebraically connected component of $Z(Q)_{[a, b]}$. If $C_{[a, b)}$ is not semi-algebraically connected, then $b$ is a pseudo-critical value of $Z(Q)$.

Proof. By Lemma 2 there exists $D$, a semi-algebraically connected component of $Z\left(Q_{2}\right)_{[a, b]}$, such that $C=\lim _{\zeta}(D)$. According to Hardt's triviality theorem, there exists $a^{\prime} \in[a, b)$, such that for every $d \in\left[a^{\prime}, b\right), C_{[a, d]}$ is not semi-algebraically connected. Hence, by Proposition $4, D_{[a, c]}$ is also not semi-algebraically connected for every $c \in \mathbf{R}\langle\zeta\rangle$ with $\lim _{\zeta}(c)=d$. Let $c \in\left[a^{\prime}, b\right] \subset \mathbf{R}\langle\zeta\rangle$ be the smallest value so that $D_{[a, c]}$ is semi-algebraically connected; then $c$ is infinitesimally close to $b$ and according to Proposition $1, c$ is a critical value of $Z\left(Q_{2}\right)$. Hence $b$ is a pseudo-critical value.

The following result appears in [13] for the case of the reals. We prove it for an arbitrary real closed field $\mathbf{R}$.

Proposition 6. Let $Z(Q) \subset B(0, M)$ be a bounded algebraic set with $[a, b] \subset$ $[-M, M]$. If $C$ is a semi-algebraically connected component of $Z(Q)_{[a, b]}$ and if $c \in(a, b)$ and $[a, b] \backslash\{c\}$ contains no pseudo-critical value of $\pi$ on $Z(Q)$, then $C_{c}$ is semi-algebraically connected.

Proof. Let $D$ be a semi-algebraically connected component of $Z\left(Q_{2}\right)_{[a, b]}$ for which $C=\lim _{\zeta}(D)$. Note that, for every $c^{\prime} \in[a, b], D_{c^{\prime}}$ is a union of semi-algebraically connected components of $Z\left(Q_{2}\right)_{c^{\prime}}$. Clearly, there exists an infinitesimal $\beta$ such that the critical values of the map $\pi$ on $Z\left(Q_{2}\right)$ in the interval $[a, b]$, if they exist, lie in the interval $[c-\beta, c+\beta]$. We claim that $D_{[c-\beta, c+\beta]}$ is semi-algebraically connected. Let $x, y$ be any two points in $D_{[c-\beta, c+\beta]}$. We will show that there exists a semi-algebraic path connecting $x, y$ lying inside $D_{[c-\beta, c+\beta]}$. Since $D$ itself is semialgebraically connected, there exists a semi-algebraic path $\gamma:[0,1] \rightarrow D$, with $\gamma(0)=x, \gamma(1)=y$, and $\gamma(t) \in D, 0 \leq t \leq 1$. If $\gamma(t) \in D_{[c-\beta, c+\beta]}$ for all $t \in[0,1]$, we are done. Otherwise, the path $\gamma$ is the union of a finite number of closed connected pieces $\gamma_{i}$, each lying either in $D_{[a, c-\beta]}, D_{[c+\beta, b]}$ or $D_{[c-\beta, c+\beta]}$. By Proposition 2 the connected components of $D_{c-\beta}$ (resp. $D_{c+\beta}$ ) are in 1-1 correspondence with the connected components of $D_{[a, c-\beta]}$ (resp. $D_{[c+\beta, b]}$ ) containing them. Thus, we can replace each of the $\gamma_{i}$ lying in $D_{[a, c-\beta]}\left(\right.$ resp. $\left.D_{[c+\beta, b]}\right)$ with end points in $D_{c-\beta}$ (resp. $D_{c+\beta}$ ), by another segment with the same end points but lying completely in $D_{c-\beta}$ (resp. $\left.D_{c+\beta}\right)$. We thus obtain a new semi-algebraic path $\gamma^{\prime}$ connecting 
$x, y$ and lying inside $D_{[c-\beta, c+\beta]}$. It is clear that $\lim _{\zeta}\left(D_{[c-\beta, c+\beta]}\right)$ coincides with $C_{c}$. Since $D_{[c-\beta, c+\beta]}$ is bounded, $C_{c}$ is semi-algebraically connected by Proposition 4.

According to Proposition 1 , if $Z(Q)$ is a smooth manifold, with $x \in Z(Q), \pi(x)=$ $c$ and for some $r, Z(Q) \cap B(x, r)_{<c}=\emptyset$, then $c$ is a critical value of $Z(Q)$. Unfortunately, and contrary to what we claimed in $[2,4]$, we do not know whether we can conclude from the existence of some $r$, such that $Z(Q) \cap B(x, r)_{<c}=\emptyset$, that $c$ is a pseudo-critical value without the hypothesis that $Z(Q)$ is a smooth hypersurface. More precisely, we do not know whether or not Lemma 1 announced in $[2,4]$ is correct. It is for this reason that we introduce the notion of a special value.

Definition 1. A special value of $Z(Q)$ is a $c \in \mathbf{R}$ for which there exists $y \in Z\left(Q_{1}\right)$ with $\lim _{\zeta}(\pi(y))=c, g(y)$ infinitesimal and $y$ a local minimum of $g$ on $Z\left(Q_{1}\right)$, where

$$
g(X)=\sum_{i=2}^{k} \frac{\partial Q_{1}{ }^{2}}{\partial X_{i}} / \sum_{i=1}^{k} \frac{\partial Q_{1}{ }^{2}}{\partial X_{i}}
$$

Note that any pseudo-critical value of $\pi$ on $Z(Q)$ is a special value of $Z(Q)$.

Proposition 7. If $Z(Q)$ is bounded and $x$ is a point of $Z(Q)_{c}$ at which $Z(Q) \cap$ $B(x, r)_{<c}$ is empty for a positive $r$, then $c$ is a special value of $Z(Q)$.

Proof. The proposition is an immediate consequence of the following two lemmas.

Lemma 3. Suppose that $Z(Q) \subset B(0, M)$ and $x$ is a point of $Z(Q)_{c}$ at which $Z(Q) \cap B(x, r)_{<c}$ is empty for some positive $r$. Then there is a point $y \in Z\left(Q_{1}\right) \cap$ $B(x, r)$ at which $\lim _{\zeta}(\pi(y))=c$ and $\lim _{\zeta}(g(y))=0$.

Lemma 4. If $y$ is a point of $Z\left(Q_{1}\right) \cap B(x, r)$ at which $\lim _{\zeta}(\pi(y))=c$ and $\lim _{\zeta}(g(y))$ $=0$, then $c$ is a special value of $Z(Q)$.

Proof of Lemma 3. Note that the statement of the lemma can be translated into a first order formula of the language of ordered fields with parameters in $R$. This becomes clear once we observe that by Proposition 4, the statement

"there exists a point $y \in Z\left(Q_{1}\right)$ such that $\lim _{\zeta}(\pi(y))=c$ and $\lim _{\zeta}(g(y))=0, "$

is equivalent to the first order formula

$$
\forall \epsilon>0 \exists \delta>0 \forall t 0<t<\delta \exists y Q_{1, t}(y)=0 \wedge g_{t}(y)^{2}+(\pi(y)-c)^{2}<\epsilon,
$$

where $Q_{1, t}$ and $g_{t}$ are the polynomials and rational functions obtained after replacing $\zeta$ by $t$ in the definitions of $Q_{1}$ and $g$. By the transfer principle, it suffices to prove the proposition over the reals $\mathbb{R}$, which is what we now proceed to do. If there is a critical value of $\pi$ on $Z\left(Q_{1}\right)$ infinitesimally close to $c$, we are done. Otherwise, suppose that there is no critical value of $\pi$ on $Z\left(Q_{1}\right)$ in an interval $(c-d, c+d)_{\mathbb{R}\langle\zeta\rangle}$ with $d \in \mathbb{R}$. We can suppose without loss of generality that $r<d$. We argue by contradiction and suppose that for every $y$ at which $Q_{1}(y)=0 \wedge \lim _{\zeta}(\pi(y))=c, g(y)$ is not infinitesimal. Since $Z(Q) \cap B(x, r)_{<c}=\emptyset$, we know that for any $y \in Z\left(Q_{1}\right) \cap B(x, r)_{\leq c}, \lim _{\zeta}(\pi(y))=c$ and thus $g(y)$ is not infinitesimal. Let $a \in \mathbb{R}$ be a positive number smaller than any value of $g$ on 
$Z\left(Q_{1}\right) \cap B(x, r)_{\leq c}$. Let

$$
U^{\prime}=\left\{t \in \mathbb{R} \mid g_{t}<a \text { on } Z\left(Q_{1, t}\right) \cap B(x, r)_{\leq c}\right\} .
$$

Let $U^{\prime \prime}$ be the set of $t \in \mathbb{R}$ such that $Z\left(Q_{1, t}\right)$ has no critical value on $(c-d, c+d)$ and $U=U^{\prime} \cap U^{\prime \prime}$. The set $U$ is semi-algebraic and its extension to $\mathbb{R}\langle\zeta\rangle$ contains $\zeta$. Thus, it contains an interval $\left(0, t_{0}\right)$ by Proposition 3 . For every $t \in\left(0, t_{0}\right)$, let $y_{t}$ be a point in $Z\left(Q_{1, t}\right) \cap B(x, r)_{\leq c}$ whose last $k-1$ coordinates coincide with the last $k-1$ coordinates of $x$ (such a point must exist because $Q_{1}$ is negative at $x$ and positive at the point $\left.\left(c-r, x_{2}, \ldots, x_{k}\right)\right)$.

Consider the curve $\gamma_{t}$ on $Z\left(Q_{1, t}\right)$ through $y_{t}$ which at each of its points is tangent to the gradient of $X_{1}$ on $Z\left(Q_{1, t}\right)$. The idea is to show that the direction of the tangent to $\gamma_{t}$ is always sufficiently close to the $X_{1}$ direction so that $\gamma_{t}$ intersects the sphere $S(x, r)$ at a point whose $X_{1}$ co-ordinate is far from $c$.

A straightforward computation proves that the gradient of $X_{1}$ on $Z\left(Q_{1, t}\right)$ at a point of $Z\left(Q_{1, t}\right)$ is proportional to

$$
G=\left(\sum_{i=2}^{k} \frac{\partial Q_{1}^{2}}{\partial X_{i}},-\frac{\partial Q_{1}}{\partial X_{1}} \frac{\partial Q_{1}}{\partial X_{2}}, \ldots,-\frac{\partial Q_{1}}{\partial X_{1}} \frac{\partial Q_{1}}{\partial X_{k}}\right) .
$$

For every point of $\gamma_{t}$, the vector $G$ thus belongs to the half-cone $\mathcal{C}$ of center $x$, based on the $k-1$-ball of radius $\sqrt{\frac{1-a}{a}}$ and center $\left(x_{1}-1, x_{2}, \ldots, x_{k}\right)$ in the hyperplane $X_{1}=x_{1}-1$. It follows that the curve $\gamma_{t}$ is completely contained in $\mathcal{C}$. Since there is no critical value of $\pi$ on $Z\left(Q_{1, t}\right)$ in $(c-d, c+d)$, the curve $\gamma_{t}$ is defined over $(c-d, c+d)$ and thus meets $S(x, r) \cap \mathcal{C}$. Since $\mathcal{C} \cap S(x, r) \cap Z\left(Q_{1, t}\right) \neq \emptyset$ is true for every $t \in\left(0, t_{0}\right)$ it follows from Proposition 3 that $\mathcal{C} \cap S(x, r) \cap Z\left(Q_{1}\right) \neq \emptyset$. Thus, taking $\lim _{\zeta}$ of the point so obtained, $B(x, r)_{<c} \cap Z(Q) \neq \emptyset$, which is a contradiction.

Proof of Lemma 4. If $g$ is zero anywhere that the first coordinate is infinitesimally close to $c$, then $c$ is a pseudo-critical value and we are done. Alternatively, we may assume that $g$ is non-zero in any slab of infinitesimal width containing $X_{1}=c$. Let $y$ be given by our hypothesis, i.e. $\lim _{\zeta}(\pi(y))=c, \lim _{\zeta}(g(y))=0$. We let $C$ be the semi-algebraically connected component of $Z\left(Q_{1}\right)$ containing $y$. Define $v$ by $\pi(y)=v$. Then $g$ attains its minimum on $C_{v}$ at some point $z \in C_{v}$. Let $t$ be this minimum. It is clear that $t$ is infinitesimal. Consider the set $A=\{w \mid$ $\left.\min _{C_{w}}(g) \leq t\right\}$. This set $A$ is closed, bounded, semi-algebraic, and thus a union of closed intervals $\left[a_{1}, b_{1}\right] \cup \ldots \cup\left[a_{h}, b_{h}\right]$ with $a_{i} \leq b_{i}<a_{i+1}$. Let $\left[a_{i}, b_{i}\right]=[a, b]$ be the interval containing $v$. If $a$ and $b$ are both infinitesimally close to $v$ take $u$ and $w$ so that $b_{i-1}<u<a=a_{i} \leq b=b_{i}<w<a_{i+1}$ with $u$ and $w$ infinitesimally close to $v$. The minimum of $g$ on $C_{[u, w]}$ occurs in the interior of the slab since it is smaller at $C_{v}$ than its minimum both on $C_{u}$ and $C_{w}$. It follows that $c$ is a special value of $Z(Q)$. Assume on the contrary that $[a, b]$ is such that $a$ or $b$ is not infinitesimally close to $v$. We are going to prove that this leads to a contradiction, namely, that in this case $Z\left(Q_{1}\right)$ is not bounded over $R$. According to Hardt's triviality theorem, there exists a family $\phi_{j}$ of smooth semi-algebraic curves parametrized by open segments $\left(\alpha_{j}, \beta_{j}\right)$ covering $(a, b)$ (with the exception of a finite number of points) such that $g\left(\phi_{j}(x)\right)$ is smaller than $t$. If $T_{j}(x)=\left(T_{j, 1}(x), \ldots, T_{j, k}(x)\right)$ is the tangent vector to 
$\phi_{j}$ at $\left(x, \phi_{j}(x)\right)$, we have

$$
\begin{gathered}
T_{j, 1} \frac{\partial Q_{1}}{X_{1}}+T_{j, 2} \frac{\partial Q_{1}}{X_{2}}+\ldots+T_{j, k} \frac{\partial Q_{1}}{X_{k}}=0 \\
-T_{j, 1} \frac{\partial Q_{1}}{X_{1}}=T_{j, 2} \frac{\partial Q_{1}}{X_{2}}+\ldots+T_{j, k} \frac{\partial Q_{1}}{X_{k}} \\
T_{j, 1}^{2} \leq \frac{t}{1-t}\left\|\left(T_{j, 2}, \ldots, T_{j, k}\right)\right\|^{2} .
\end{gathered}
$$

Thus, at every point on each of these curves, $\left|\frac{T_{j, 1}(x)}{T_{j, i}(x)}\right|<\sqrt{\frac{k t}{1-t}}=t^{\prime}$ for some $2 \leq i \leq k$. Hence, we can suppose - subdividing further if needed and producing more curves - that on each of these curves, $\left|\frac{T_{j, 1}(x)}{T_{j, i}(x)}\right|<t^{\prime}$ for some $2 \leq i \leq k$. Let $N$ be the number of the curves so obtained. We prove now that the interval $\left(v, v+2 M N t^{\prime}\right)$ contains $w$ such that $\min _{C_{w}}(g)>t$. Suppose on the contrary that at every value $w \in\left(v, v+2 M N t^{\prime}\right)$, $\min _{C_{w}}(g) \leq t$. Then there is an interval of length at least $2 M t^{\prime}$ over which the curve $\phi_{j}(x)$ is smooth and $\left|\frac{T_{j, 1}(x)}{T_{j, i}(x)}\right|$ is less than $t^{\prime}$. It follows from the mean value theorem that the projection of this curve on the $X_{i}$ axis is bigger than $2 M$, which contradicts the fact that $C \subset B(0, M)$. Similarly, the interval $\left(v-2 M N t^{\prime}, v\right)$ contains $u$ such that $\min _{C_{u}}(g)>t$. Note that both $u$ and $w$ are infinitesimally close to $c$. This contradicts the fact that $a$ or $b$ is not infinitesimally close to $v$ and concludes the argument.

Consider the algebraic set $Z$ defined by the $k+1$ polynomial equations in $k+1$ variables $\left(X_{1}, \ldots, X_{k}, \lambda\right)$ which are obtained by removing the denominators from the following equations: $Q_{1}=0, \frac{\partial Q_{1}}{\partial X_{1}}=\lambda \frac{\partial g}{\partial X_{1}}, \ldots, \frac{\partial Q_{1}}{\partial X_{k}}=\lambda \frac{\partial g}{\partial X_{k}}$. The local minima of $g$ on $Z\left(Q_{1}\right)$ are contained in the projection of $Z$ to the first $k$ coordinates. A consequence of the proof of Lemma 4 is the following.

Corollary 1. If $C^{\prime}$ is a semi-algebraically connected component of $Z$ on which $g$ has an infinitesimal local minimum on $Z\left(Q_{1}\right)$, then $\lim _{\zeta}\left(\pi\left(C^{\prime}\right)\right)$ is a single point.

Proof. Let $x$ be a point of $C^{\prime}$ where $g$ has an infinitesimal local minimum on $Z\left(Q_{1}\right)$ and let $v=g(x)$. Note that $g$ is constant on $C^{\prime}$. The projection of $C^{\prime}$ on the $X_{1}$-axis, $\pi\left(C^{\prime}\right)$, is contained in $A=\left\{w \mid \min _{C_{w}}(g) \leq v\right\}$ where $C$ is the semi- algebraically connected component of $Z\left(Q_{1}\right)$ containing $x$. Since $\pi\left(C^{\prime}\right)$ is semi-algebraically connected, following the proof of Lemma 4 we see that $\pi\left(C^{\prime}\right)$ is contained in an infinitesimal segment.

Let $S$ be a basic, closed, bounded semi-algebraic set defined as

$$
S=\left\{x \in \mathbf{R}^{k} \mid \forall P \in \mathcal{P}, P(x) \geq 0\right\},
$$

where the polynomials $P \in \mathcal{P}$ are such that $Z(P)$ is bounded. A special value of $S$ is defined to be a special value of a $Z\left(\mathcal{P}^{\prime}\right)$ where $\mathcal{P}^{\prime} \subset \mathcal{P}$. The following two lemmas play a crucial role in the proof of the correctness of the Roadmap Algorithm. If $B$ is a semi-algebraic set, we denote by $\bar{B}$ its closure in the Euclidean topology. 
Lemma 5. Let $C$ be a semi-algebraically connected component of $S_{[a, c]}$, and let $B_{1}, \ldots, B_{h}$ be the semi-algebraically connected components of $C_{[a, c)}$. Then $C=$ $C_{1} \cup \ldots C_{h} \cup C_{h+1} \cup \ldots \cup C_{N}$ where $C_{i}=\bar{B}_{i}$ for $1 \leq i \leq h$ and for $j>h, C_{j}$ is a semi-algebraically connected component of $Z\left(\mathcal{P}_{j}\right) \cap C_{c}$ where $\mathcal{P}_{j} \subset \mathcal{P}$.

Proof. If $x \in C$ and $x \notin \bigcup_{1 \leq i \leq h} \bar{B}_{i}$, then there is a positive $r$ such that $C \cap$ $B(x, r)_{<c}=\emptyset$. If $\mathcal{P}^{\prime}$ is the set of polynomials in $\mathcal{P}$ which vanish at $x$, then $Z\left(\mathcal{P}^{\prime}\right) \cap$ $B(x, r)_{<c}=\emptyset$. Hence, $c=\pi(x)$ is a special value of $Z\left(\mathcal{P}^{\prime}\right)$ by Proposition 7 . It is clear that the semi-algebraically connected component of $Z\left(\mathcal{P}^{\prime}\right) \cap C_{c}$ containing $x$ is contained in $C$.

Using the same notation, we have:

Lemma 6. If there is an $i, j \in\{1, \ldots, N\}$ such that $C_{i} \cap C_{j} \neq \emptyset$, then $c$ is a special value of some $Z\left(\mathcal{P}^{\prime}\right)$ where $Z\left(\mathcal{P}^{\prime}\right) \cap C_{i} \cap C_{j} \neq \emptyset$ and $\mathcal{P}^{\prime} \subset \mathcal{P}$.

Proof. The statement is clearly true in the case $i>h$ or $j>h$ by Lemma 5 . Suppose, without loss of generality, that $C_{i}=\bar{B}_{1}, C_{j}=\bar{B}_{2}, \bar{B}_{1} \cap \ldots \cap \bar{B}_{I} \neq \emptyset$ and $1, \ldots, I$ is a maximal family with this property. Let $x$ be a point of this intersection. Clearly, $x$ belongs to the boundary of $S$ and the set, $\mathcal{P}^{\prime} \subset \mathcal{P}$, of polynomials in $\mathcal{P}$ which vanish at $x$ is not empty. According to Hardt's triviality theorem there is $w \in[a, c)$ such that $Z\left(\mathcal{P}^{\prime}\right)_{[w, c)}$ is semi-algebraically homeomorphic to $Z\left(\mathcal{P}^{\prime}\right)_{w} \times[w, c)$ and $C_{[w, c)}$ is semi-algebraically homeomorphic to $C_{w} \times[w, c)$. Let $D$ be the connected component of $Z\left(\mathcal{P}^{\prime}\right)_{[w, c]}$ containing $x$. We consider two cases according to whether or not $D_{w}$ is empty: If $D_{w}$ is empty, then $c$ is a special value of $Z\left(\mathcal{P}^{\prime}\right)$ by Proposition 7 .

If $D_{w}$ is not empty, then some semi-algebraically connected component of $C_{[a, c)}$ intersects $Z\left(\mathcal{P}^{\prime}\right)$ in every neighborhood of $x$. Suppose, without loss of generality, that it is $B_{1}$. Consider a maximal subset of $\mathcal{P}$, say $\mathcal{P}^{\prime \prime}$, such that $Z\left(\mathcal{P}^{\prime \prime}\right)$ intersects $B_{2}$ in every neighborhood of $x$. The set $\mathcal{P}^{\prime \prime}$ is non-empty and contained in $\mathcal{P}^{\prime}$. According to Hardt's triviality theorem there is a $w^{\prime} \geq w$ such that $Z\left(\mathcal{P}^{\prime \prime}\right)_{\left[w^{\prime}, c\right)}$ is semi-algebraically homeomorphic to $Z\left(\mathcal{P}^{\prime \prime}\right)_{w^{\prime}} \times\left[w^{\prime}, c\right)$. Let $Z$ be the semi-algebraically connected component of $Z\left(\mathcal{P}^{\prime \prime}\right)_{\left[w^{\prime}, c\right]}$ containing $x$. By the maximality of $Z\left(\mathcal{P}^{\prime \prime}\right)$, there is a connected component $Z_{1}$ of $Z_{\left[w^{\prime}, c\right)}$ contained in $B_{2\left[w^{\prime}, c\right)}$. Since $Z\left(\mathcal{P}^{\prime}\right) \subset Z\left(\mathcal{P}^{\prime \prime}\right)$ and $Z\left(\mathcal{P}^{\prime}\right)_{\left[w^{\prime}, c\right)}$ meets $B_{1}, Z\left(\mathcal{P}^{\prime \prime}\right)_{\left[w^{\prime}, c\right)}$ is not semialgebraically connected. We conclude by Proposition 5 that $c$ is a pseudo-critical value, and hence a special value, of $Z\left(\mathcal{P}^{\prime \prime}\right)$.

\section{Algorithmic tools}

Sign Determination Subroutine. The input is a Gröbner basis for a zerodimensional ideal $I$ for which the quotient has dimension $O(d)^{k}$, together with a list of $s$ polynomials $\mathcal{P}$ whose degrees are all at most $d$. The output is the list of non-empty sign-conditions on the family $\mathcal{P}$ at the zeroes of $I$. The complexity of this algorithm is $s d^{O(k)}$ (see [1], section 3.1.4, page 1029).

Thom encoding. Let $f \in \mathbf{R}[X]$ and $\sigma \in\{0,1,-1\}^{\operatorname{deg}(f)}$; then $\mathcal{R}(\sigma)=\{x \in \mathbf{R} \mid$ $\left.\operatorname{sign} f^{(i)}(x)=\sigma(i)\right\}$ is either a point, or an open interval, or empty ([5], page 37). If $\mathcal{R}(\sigma)$ is not empty, $\sigma$ is the Thom encoding of the set $R(\sigma)$. If $x$ is a root of $f$, and $\sigma$ is the list of the signs of the derivatives of $f$ at $x$ (so that $\sigma(0)=0$ ), $\mathcal{R}(\sigma)=\{x\}$ and $\sigma$ distinguishes $x$ from all the other roots of $f$. The ordering of the roots can also be recovered from the Thom encodings [10]. The Thom encodings of 
the roots of a univariate polynomial $f$ are computed using the Sign Determination Subroutine with input $f$ and its derivatives. The complexity of this computation is $\tau^{O(1)}$ where $\tau$ is the degree of $f$.

Univariate representation. A $k$-univariate representation is a $k+2$-tuple

$$
u=\left(f(T), g_{0}(T), g_{1}(T), \ldots, g_{k}(T)\right),
$$

of elements of $\mathbf{R}[T]$ where $\operatorname{deg}\left(g_{i}\right) \leq \operatorname{deg}(f)$. The point $p=\left(x_{1}, x_{2}, \ldots, x_{k}\right)$ in $\mathbf{R}[i]^{k}$ is associated to $u$ if there exists a root $t$ of $f(T)$ in $\mathbf{R}[i]$ such that $x_{i}=g_{i}(t) / g_{0}(t)$, for $i=1, \ldots, k$.

Real univariate representation. A real $k$-univariate representation is a pair $(u, \sigma)$ where $u$ is a $k$-univariate representation

$$
u=\left(f(T), g_{0}(T), g_{1}(T), \ldots, g_{k}(T)\right)
$$

and $\sigma \in\{-1,0,1\}^{\operatorname{deg}(f)}$ is the Thom encoding of a root $t_{\sigma}$ of $f(T)$ in $\mathbf{R}$. The point $p=\left(x_{1}, x_{2}, \ldots, x_{k}\right)$ in $\mathbf{R}^{k}$ is associated to $(u, \sigma)$ if $x_{i}=g_{i}\left(t_{\sigma}\right) / g_{0}\left(t_{\sigma}\right)$, for $i=1, \ldots, k$, and $(u, \sigma)$ represents $p$.

Projection Subroutine. The input is a univariate representation

$$
u=\left(f, g_{0}, \ldots, g_{k}\right) .
$$

The output of the algebraic stage is a univariate polynomial $h$ whose roots are $g_{1}(t) / g_{0}(t)$ for all $t$ which are roots of $f$ in $\mathbf{R}[i]$. The output of the sign determination stage are the Thom encodings of the real roots of $f$ and of the corresponding real roots of $h$.

The resultant $h(Y)$ of $h_{1}(Y, T)=f(T)$, and $h_{2}(Y, T)=Y g_{0}(T)-g_{1}(T)$ with respect to $T$, is a polynomial in $Y$ whose roots are the values of $g_{1}(t) / g_{0}(t)$ where $t$ is a root of $f$. The algebraic stage of the subroutine computes $h$.

In the sign determination stage of the subroutine, we first compute the Thom encoding of the real roots of $f$. Then, for every Thom encoding $\sigma$ of a root $t_{\sigma}$ of $h$, we compute the Thom encoding of the root of $h$ which is equal to $g_{1}\left(t_{\sigma}\right) / g_{0}\left(t_{\sigma}\right)$ using the Sign Determination Subroutine. If $\tau$ is a bound on the degree of $f$, the complexity of the Projection Subroutine is $\tau^{O(1)}$.

Cell Representative Subroutine. The input is a polynomial $Q$ of degree at most $d$ in $k$ variables and the output is a set of $k$-univariate representations of degree at most $O(d)^{k}$. The points associated to these $k$-univariate representations meet every semi-algebraically connected component (or cell) of $Z(Q)$. The complexity of this subroutine is $d^{O(k)}$ ([1], section 3.1.2, page 1026).

Special Values Subroutine. The input is a polynomial $Q$ in $\mathbf{R}\left[X_{1}, \ldots, X_{k}\right]$ of degree at most $d$ and the output of the algebraic stage is a set of univariate polynomials of degree at most $d^{O(k)}$ whose roots in $\mathbf{R}$ contain the special values of $Z(Q)$. In the sign determination phase we compute the Thom encoding of the roots in $\mathbf{R}$ of the polynomials output by the algebraic stage. The description of the subroutine follows: the set $Z \subset Z\left(Q_{1}\right) \times \mathbf{R}\langle\zeta\rangle$ is the algebraic set defined by the $k+1$ polynomial equations in $k+1$ variables $\left(X_{1}, \ldots, X_{k}, \lambda\right)$ obtained by removing the denominators from the following equations:

$$
Q_{1}=0, \frac{\partial Q_{1}}{\partial X_{1}}=\lambda \frac{\partial g}{\partial X_{1}}, \ldots, \frac{\partial Q_{1}}{\partial X_{k}}=\lambda \frac{\partial g}{\partial X_{k}} .
$$


We use the Cell Representative Subroutine to find a set of $k$-univariate representations with associated real points meeting every connected component of $Z$. In the algebraic stage of the subroutine, we compute the polynomial $h$ having as zeroes the $\lim _{\zeta}$ of the first coordinates of these points (using the algebraic stage of the Projection Subroutine and the algebraic $\lim _{\zeta}$ process in [20]). In the sign determination stage of the subroutine, we compute the Thom encodings of the zeroes of $h$. The complexity of this routine is $d^{O(k)}$.

Sample Points on a Variety Subroutine. The input is a set of $s$ polynomials, $\mathcal{P} \subset \mathbf{R}\left[X_{1}, \ldots, X_{k}\right]$, each of degree at most $d$ and $Q \in \mathbf{R}\left[X_{1}, \ldots, X_{k}\right]$ of degree at most $d$, with the variety $Z(Q)$ of real dimension $k^{\prime}$. The output is a set of $s^{k^{\prime}} O(d)^{k} k$-univariate representations such that the set of points associated to these $k$-univariate representations meet every semi-algebraically connected component of every non-empty sign condition of $\mathcal{P}$ on $Z(Q)$. This subroutine has complexity $s^{k^{\prime}+1} d^{O(k)}[3]$.

Parametrized univariate representations. A parametrized $k$-univariate representation with parameter $Y$ is a $k+2$-tuple

$$
u=\left(f(Y, T), g_{0}(Y, T), g_{1}(Y, T), \ldots, g_{k}(Y, T)\right),
$$

of polynomials in $\mathbf{R}[Y, T]$ where the degrees of the $g_{i}$ in $T$ are not greater than the degree of $f$ in $T$.

Parametrized Cell Representatives Subroutine. The input is a polynomial $Q\left(Y, X_{1}, \ldots, X_{k}\right)$ of degree $d$ with coefficients in $\mathbf{R}$ and the output is a set of parametrized $k$-univariate representations $\mathcal{U}$. For each specialization $y$, of the parameter $Y$, the set of points associated with the $k$-univariate representations $u(y)$ for $u \in \mathcal{U}$ is a subset of the algebraic set defined by $Q\left(y, X_{1}, \ldots, X_{k}\right)=0$ and meets every semi-algebraically connected component of $Z(Q)_{y}$. There are $d^{O(k)} k$-univariate representations output and the degree of each polynomial in these representations is $O(d)^{k}$. The subroutine is a special case of the Parametrized Cell Representatives Subroutine in [1] (section 5.1.2, page 1035) in which $\ell=1$, i.e. there is only one parameter $Y$. The complexity of the subroutine is $d^{O(k)}$.

Real univariate representations over an interval $(a, b)$. A real $k$-univariate representation over $(a, b)$ is specified by $(A, \alpha, B, \beta, u, \sigma)$ where

- $a$ and $b$ are roots of the univariate polynomials $A$ and $B$ with Thom encodings $\alpha$ and $\beta$,

- $u=\left(f(Y, T), g_{0}(Y, T), g_{1}(Y, T), \ldots, g_{k}(Y, T)\right)$ is a parametrized $k$-univariate representation,

- $\sigma \in\{-1,0,1\}^{\operatorname{deg}(f)}$ is a sign condition such that for every $y \in(a, b)$ there exists a real root $t_{\sigma}(y)$ of $f(y, T)$ with Thom encoding $\sigma$.

To a real $k$-univariate representation over $(a, b)$ is associated a curve segment parametrized along the $Y$ axis, i.e. a continuous semi-algebraic function from $(a, b)$ to $\mathbf{R}^{k}$ : to each point $y$ of $(a, b)$ is associated the point of $\mathbf{R}^{k}$ with coordinates

$$
\left(\frac{g_{1}\left(y, t_{\sigma}(y)\right)}{g_{0}\left(y, t_{\sigma}(y)\right)}, \ldots, \frac{g_{k}\left(y, t_{\sigma}(y)\right)}{g_{0}\left(y, t_{\sigma}(y)\right)}\right) .
$$


Real univariate representations over a point $c$. A real $k$-univariate representation of $(A, u)$ where $A$ is a univariate polynomial and $u$ is a parametrized univariate representation with parameter $Y$ is specified by $(A, \alpha, u, \sigma)$ where

- $\alpha$ is the Thom encoding of a root $c$ of the univariate polynomial $A$,

- $\sigma \in\{-1,0,1\}^{\operatorname{deg}(f)}$ is a sign condition such that there exists a real root $t_{\sigma}$ of $f(c, T)$ with Thom encoding $\sigma$.

To the real univariate representation over $c(A, \alpha, u, \sigma)$, is associated the point of $\mathbf{R}^{k+1}$ with coordinates

$$
\left(c, \frac{g_{1}\left(c, t_{\sigma}\right)}{g_{0}\left(c, t_{\sigma}\right)}, \ldots, \frac{g_{k}\left(c, t_{\sigma}\right)}{g_{0}\left(c, t_{\sigma}\right)}\right) .
$$

Intersection of a real univariate representation over $(a, b)$ with a hyperplane $Y=c$. Given a real univariate representation $(u, \sigma)$ over $(a, b)$ and a point $c$ of $(a, b)$ described by a univariate polynomial $A(Y)$ the Thom encoding $\alpha$ of the root $c$ of $A$, the intersection of $(u, \sigma)$ with the hyperplane $Y=c$ is the real univariate representation over $c$ given by $(A, \alpha, u, \sigma)$.

Partition of the $Y$-axis associated to a family $\mathcal{A}$ of univariate polynomials. The partition of the $Y$-axis associated to $\mathcal{A}$ is the ordered list $y_{1}<\ldots<y_{n}$ of the roots of the polynomials of $\mathcal{A}$ each $y_{i}$ being specified by a couple $\left(A_{i}, \alpha_{i}\right)$ where $A_{i} \in \mathcal{A}, A_{i}\left(y_{i}\right)=0$ and $\alpha_{i}$ is the Thom encoding of $y_{i}$. The computation of the partition associated to a family $\mathcal{A}$ of at most $s$ polynomials of degree at most $d$ is done using the Sign Determination Subroutine in time $s \log s d^{O(1)}$.

Parametrized Real Roots Subroutine. The input is a parametrized $k$-univariate representation with parameter $Y$

$$
u=\left(f(Y, T), g_{0}(Y, T), g_{1}(Y, T), \ldots, g_{k}(Y, T)\right)
$$

and the output is a family $\mathcal{A}_{u} \subset \mathbf{R}[Y]$, with the property that for each $y$ the signs of the polynomials in $\mathcal{A}_{u}$ at $y$ determine the number of complex roots of $f(y, T)$, the number of real roots of $f(y, T)$, the Thom encodings of the real roots of $f(y, T)$, as well as the degrees of $f(y, T)$ and of $\operatorname{gcd}\left(f(y, T), f^{\prime}(y, T)\right)$. The family $\mathcal{A}_{u}$ consists of the principal subresultant coefficients of $f(Y, T)$ and each of its successive derivatives with respect to $T$, defining a partition of the $Y$-axis and a finite set of real $k$-univariate representations over intervals and points of this partition. The union of the curve segments and points associated are the points $g_{i}(t, y) / g_{0}(t, y)$ for $y$ and $t$ in $\mathbf{R}$ with $f(y, t)=0$.

Algebraic Stage: Compute the finite set $\mathcal{A}_{u} \subset \mathbf{R}[Y]$ consisting of the principal subresultant coefficients of $f(Y, T)$ and each of its successive derivatives with respect to $T$.

Sign Determination Stage: The input is the family $\mathcal{A}_{u}$ and the output is the partition of the $Y$-axis associated to $\mathcal{A}_{u}$. Over each open interval and point of this partition compute the signs of the polynomials $\mathcal{A}_{u}$. Then compute for each interval and point of the partition the list of Thom encodings of the real roots of the polynomial $f(Y, T)$, outputting real univariate representations over segments and points of the partition. This is done using the Sign Determination Subroutine.

If the parametrized univariate representation is given by polynomials of degree bounded by $D$, the complexity of the Real Parametrized Univariate Representation Subroutine is $D^{O(1)}$. 
Limit of a Curve Segment Subroutine. The input is a real $k$-univariate representation over $(a, b)$ with coefficients in $\mathbf{R}[\varepsilon], v(\varepsilon)=\left(A(\varepsilon), \sigma_{a}, B(\varepsilon), \sigma_{b}, u(\varepsilon), \sigma\right)$, such that the curve segment associated is bounded over $\mathbf{R}$. Its output is the "limit of the curve segment when $\epsilon$ tends to 0 ", i.e.: a partition of $\left[\lim _{\epsilon}(a), \lim _{\epsilon}(b)\right]$ into a finite number of open intervals and points, and a real $k$-univariate representation over these intervals and points such that the union of the curve segments and points associated is the image under $\lim _{\epsilon}$ of the original curve segment.

The subroutine is a parametrized version of the Detect Bounded Roots Algorithm in [20]. If the input polynomials have degrees at most $D$, the complexity of this subroutine is $D^{O(1)}$.

\section{Curve Segments Subroutine}

The Curve Segments Subroutine is the basic building block in our algorithms. Its input is

1. a polynomial $Q \in \mathbf{R}\left[Y, X_{1}, \ldots, X_{k}\right]$, for which $Z(Q)$ is bounded and whose degree is at most $d$,

2. a set $\mathcal{M}$ of $m k+1$-univariate representations $u$ with $\operatorname{deg} u \leq \tau$, with associated points in $Z(Q)$.

The output is

1. a finite set $\mathcal{U}_{1}$ of parametrized univariate representations with parameter $Y$,

2. a family of univariate polynomials $\mathcal{L}_{1}$. The real zeroes of the polynomials in $\mathcal{L}_{1}$ are the distinguished values,

3. a finite set of real univariate representations over the intervals and points of the partition associated to $\mathcal{L}_{1}$,

4. a finite set $\mathcal{M}_{1}$ of pairs $(L, u)$ with $L \in \mathcal{L}_{1}$ and $u$ a parametrized univariate representation with parameter $Y$ and their real univariate representations.

The curve segments and points associated to the real univariate representations output are contained in $Z(Q)$ and satisfy the following properties.

$\mathrm{CS}_{1}$ : For every $y \in \mathbf{R}$ the set of curve segments and points intersect every semi-algebraically connected component of $Z(Q)_{y}$.

$\mathrm{CS}_{2}$ : For each curve segment over an interval of the partition, there exists a point over each extremity of the interval which belongs to the closure of the curve segment.

$\mathrm{CS}_{3}$ : The real zeroes of $\mathcal{L}_{1}$ contain the special values of $Z(Q)$ and the first coordinates of the real points associated to $\mathcal{M}$.

The adjacency relations between curve segments and points are also output in the subroutine.

\section{Curve Segments Subroutine.}

\section{Algebraic Stage:}

1. Call the Parametrized Cell Representatives Subroutine, with input $Q\left[Y, X_{1}, \ldots, X_{k}\right]$ to obtain $\mathcal{U}_{1}$.

2. For each parametrized $k$-univariate representation in $\mathcal{U}_{1}$, call the algebraic stage of the Parametrized Real Roots Subroutine. Call the algebraic stage of the Special Values Subroutine to obtain a univariate polynomial whose zeroes contain the Special Values. Call the algebraic stage of the Projection Subroutine to obtain the polynomials having as zeroes the 
first coordinates of the points associated to $\mathcal{M}$. Collect all the univariate polynomials so obtained in $\mathcal{L}_{1}$.

3. Place the pairs $(L, u)$ with $L \in \mathcal{L}_{1}$ and $u \in \mathcal{U}_{1}$ in a set $\mathcal{M}_{1}$. Add to $\mathcal{M}_{1}$ the pairs $\left(h,\left(f, g_{0}, g_{2}, \ldots, g_{k}\right)\right)$ for $u=\left(f, g_{0}, g_{1}, g_{2}, \ldots, g_{k}\right)$ in $\mathcal{M}$ and $h$ the projection of $u$.

\section{Sign Determination Stage:}

1. Call the sign determination stage of the Parametrized Real Roots Subroutine.

2. Compute the partition associated to $\mathcal{L}_{1}$, and intersect the real univariate representation over intervals already obtained by hyperplanes $Y=c$ with $c$ a zero of $\mathcal{L}_{1}$.

3. Determine adjacencies between curves and points, by using the Thom encodings of the real univariate representations over intervals and points.

4. Sort the set of distinguished values in increasing order.

5. Compute the real univariate representions of the elements of $\mathcal{M}_{1}$. The real points associated are called the distinguished points.

4.1. Correctness of the Curve Segments Subroutine. To verify Property $\mathrm{CS}_{1}$, we use the properties of the the output of the Parametrized Cell Representative Subroutine. By the correctness of the Parametrized Cell Representative Subroutine, we have that for every $y \in \mathbf{R}$ the union of the set of points associated to the real univariate representations $u$, over the interval containing $y$, intersects every semi-algebraically connected component of the algebraic set $Z(Q(y, X))$. To verify Property $\mathrm{CS}_{2}$, note that the algorithm outputs a set of real univariate representations over all the intervals and points of the partition. Consider any open interval $\left(a_{i}, a_{i+1}\right)$ of the partition, as well as a curve segment represented by $(u, \sigma)$ over this interval, where $u=\left(f(Y, T), g_{0}(Y, T), \ldots, g_{k}(Y, T)\right)$. Now, over the point $a_{i}$ of the partition, the subroutine outputs all points represented by $\left(u, \sigma^{\prime}\right)$ for every $\sigma^{\prime}$ corresponding to real roots of the polynomial $f\left(a_{i}, T\right)$. Since the curve segment represented by $(u, \sigma)$ is bounded and corresponds to some real root of $f\left(\frac{a_{i}+a_{i+1}}{2}, T\right)$, it is clear that one of the points represented by $\left(u, \sigma^{\prime}\right)$ belongs to the closure of this curve.

4.2. Complexity of the Curve Segments Subroutine. The total complexity of the subroutine is $m \log m \tau^{O(1)} d^{O(k)}$, using the complexities of the various subroutines involved.

\section{RoAdmap Algorithm FOR AN ALGEBRAIC SET}

We construct a roadmap of an algebraic set, but for technical reasons (in order to ensure the connectivity of the roadmap obtained) we shall construct the roadmap of an algebraic set passing through a finite number of input points.

5.1. Roadmap algorithm for a bounded algebraic set. We first describe an algorithm for the case of a bounded algebraic set. The input is

1. a polynomial $Q \in \mathbf{R}\left[X_{1}, \ldots, X_{k}\right]$ whose total degree is at most $d$ for which $Z(Q)$ is bounded,

2. a set $\mathcal{M}$ consisting of $m k$-univariate representations defined by polynomials of degree at most $\tau$. 
The algorithm is as follows. Call the Curve Segments Subroutine with $X_{1}$ as parameter. In every hyperplane defined by $X_{1}=a$ where $a$ is a distinguished value, make a recursive call to the algorithm in $\mathbf{R}^{k-1}=\pi^{-1}(a)$, with the following input: the polynomial $Q\left(a, X_{2}, \ldots, X_{k}\right)$, and the pairs $(A, u) \in \mathcal{M}_{1}$ with associated real points belonging to the fiber $\pi^{-1}(a)$.

The output, denoted $R(Z(Q), \mathcal{M})$, is a roadmap which contains the points associated to $\mathcal{M}$.

Remark 1. Note that $a$ is a root in $\mathbf{R}$ of a polynomial $A$ whose degree is either $\tau$ or $d^{O(k)}$. The polynomial $Q\left(a, X_{2}, \ldots, X_{k}\right)$ has its coefficients in the ring $\mathbf{D}[a]=\mathbf{D}[Y] / A(Y)$ rather than in $\mathbf{D}$ which is the ring generated by the coefficients of $Q$ and of the polynomials defining the $m$ real $k$-univariate representations in $\mathcal{M}$. Thus, the computations in the next recursive call take place over the ring $\mathbf{D}[a]$ rather than in $\mathbf{D}$. The algebraic part of the computation depends only on $A$ while the sign determinations process takes into account the Thom encoding $\alpha$ of $a$. Since the complexity of our algorithms is defined as the number of arithmetic operations (including comparisons) in the ring $\mathbf{D}$, we take into account the extra cost of doing arithmetic operations in rings of the form $\mathbf{D}\left[a_{1}\right], \ldots, \mathbf{D}\left[a_{1}, \ldots, a_{k-1}\right]$ when analyzing the complexity.

5.1.1. Correctness of the algorithm. Abusing terminology we shall from now on use a geometric language to describe the output of our algorithms, keeping in mind the specific form of their description.

Let $R(Z(Q), \mathcal{M})$ be the semi-algebraic set computed by our algorithm in the bounded case. We prove that $R(Z(Q), \mathcal{M})$ satisfies the two roadmap conditions RM1 and RM2. The proof is by induction on dimension. In the case of dimension one, the roadmap properties are obviously true for the set we compute. Now assume that the algorithm computes a correct roadmap for all dimensions less than $k$. That $R(Z(Q), \mathcal{M})$ satisfies condition RM2 follows from property $\mathrm{CS}_{1}$ of the Curve Segments Subroutine. That $R(Z(Q), \mathcal{M})$ satisfies condition RM1 is the content of the following two lemmas.

Lemma 7. If $c \in(a, b)$ is a distinguished value such that $[a, b] \backslash\{c\}$ contains no distinguished value of $\pi$ on $Z(Q)$ and $C$ is a semi-algebraically connected component of $Z(Q)_{[a, b]}$, then $R(Z(Q), \mathcal{M}) \cap C$ is semi-algebraically connected.

Proof. Since $[a, b] \backslash\{c\}$ contains no pseudo-critical value of the algebraic set $Z(Q)$, we know, by Proposition 6 , that $C_{c}$ is semi-algebraically connected. Moreover, by property $\mathrm{CS}_{2}$ of the output of the Curve Segment Subroutine we know that any curve segment in $R(Z(Q), \mathcal{M}) \cap C$ has an end point in $R(Z(Q), \mathcal{M})_{c}$. All these end points are in the same semi-algebraically connected component of $C_{c}$, since $C_{c}$ is semi-algebraically connected. The algorithm makes a recursive call at every distinguished value and hence at $c$. The input to the recursive call is the algebraic set $Z(Q)_{c}$ and the $k$-univariate representations of all distinguished points which include the end points of the curves in $R(Z(Q), \mathcal{M}) \cap C$. Hence, by the inductive hypothesis they are connected by the roadmap algorithm in the slice. Therefore, $R(Z(Q), \mathcal{M}) \cap C$ is semi-algebraically connected.

Lemma 8. If $C$ is a semi-algebraically connected component of $Z(Q)$, then $R(Z(Q), \mathcal{M}) \cap C$ is semi-algebraically connected. 
Proof. Let $x, y$ be two points of $R(Z(Q), \mathcal{M}) \cap C$, and let $\gamma$ be a semi-algebraic path in $C$ from $x$ to $y$. We shall use $\gamma$ to construct a path from $x$ to $y$ in $R(Z(Q), \mathcal{M}) \cap C$. Let $\left\{v_{1}<v_{2}<\cdots<v_{n}\right\}$ be the set of distinguished values and choose $u_{i}$ such that $u_{1}<v_{1}<u_{2}<v_{2}<\cdots<u_{n}<v_{n}<u_{n+1}$. There exists a finite number of points of $\gamma, x=x_{0}, x_{1}, \ldots, x_{N+1}=y$, and semi-algebraic paths $\gamma_{i}$ from $x_{i}$ to $x_{i+1}$ such that:

1. $\gamma=\bigcup_{0 \leq i \leq N} \gamma_{i}$.

2. $\gamma_{i} \subset C_{\left[u_{\ell(i)}, u_{\ell(i)+1}\right]}$.

Let $C_{i}$ be the semi-algebraically connected component of $C_{\left[u_{\ell(i)}, u_{\ell(i)+1}\right]}$ containing $\gamma_{i}$. Since $C_{i} \cap C_{i+1}$ is a finite union of semi-algebraically connected components of $C_{\pi\left(x_{i+1}\right)}, R(Z(Q), \mathcal{M}) \cap C_{i} \cap C_{i+1}$ is not empty. Choose $y_{0}=x, \ldots, y_{i} \in$ $R(Z(Q), \mathcal{M}) \cap C_{i} \cap C_{i+1}, \ldots, y_{N+1}=y$. Then $y_{i}$ and $y_{i+1}$ are in the same semialgebraically connected component of $R(Z(Q), \mathcal{M}) \cap C$ by Lemma 7 .

Remark 2. Note that it is unimportant here that the set of distinguished values contains special values, we only need it to contain pseudo-critical values. So if we are interested only in the roadmap of an algebraic set we can simplify slightly the Curve Segments Subroutine and compute only pseudo-critical values in Step 4. The special values are important in section 6 for the correctness of the Uniform Roadmap Algorithm.

5.1.2. Complexity analysis. The cost of a call to the Curve Segments Subroutine is $m \log m \tau^{O(1)} d^{O(k)}$ arithmetic operations in the ring $\mathbf{D}$. We make recursive calls at $m+d^{O(k)}$ distinguished values. Note that in a recursive call at depth $\ell$ the computations are performed over a ring $\mathbf{D}\left[a_{1}, \ldots, a_{\ell}\right]$, and a single arithmetic operation in $\mathbf{D}\left[a_{1}, \ldots, a_{\ell}\right] \operatorname{costs} \tau^{O(1)} d^{O(k \ell)}$ operations in $\mathbf{D}$. Thus, the total number of arithmetic operations made in the ring $\mathbf{D}$ during the algorithm for the bounded case is bounded by $m \log m \tau^{O(1)} d^{O\left(k^{2}\right)}$.

5.2. Roadmap algorithm in the unbounded case. Next we show how to modify the algorithm presented above to handle the case when the input algebraic set $Z(Q)$ is not bounded. If $Z(Q)$ is not bounded, we first introduce a new variable $X_{k+1}$ and a new variable $\Omega$ and replace $Q$ by the polynomial

$$
Q_{\Omega}=Q^{2}+\left(X_{1}^{2}+\cdots+X_{k+1}^{2}-\Omega^{2}\right)^{2} .
$$

We identify the real $k$-univariate representations of $\mathcal{M}$ with real $k+1$-univariate representations of $\mathcal{M}$ by setting the last coordinate equal to $O$.

Note that the algebraic set $Z\left(Q_{\Omega}\right)$ is bounded in $\mathbf{R}^{k+1}\langle 1 / \Omega\rangle$. The roadmap algorithm outputs a roadmap $R\left(Z\left(Q_{\Omega}\right), \mathcal{M}\right)$, composed of points and curves whose descriptions involve $\Omega$. Let $\mathcal{L}$ be the set of all polynomials in $\mathbf{D}[\Omega]$ whose signs were determined in the various calls to the Sign Determination Subroutine inside the algorithm. We recall from [1] (section 2.6) the following definition.

Definition 2. Given a polynomial $P=c_{q} X^{q}+\cdots+c_{p} X^{p}, q>p, c_{q}, c_{p} \neq 0$,

$$
\begin{aligned}
& C(P)=\left(\sum_{p \leq i \leq q}\left(\frac{c_{i}}{c_{q}}\right)^{2}\right)^{1 / 2}, \\
& c(P)=\left(\sum_{p \leq i \leq q}\left(\frac{c_{i}}{c_{p}}\right)^{2}\right)^{-1 / 2} .
\end{aligned}
$$


Given a set of polynomials $\mathcal{L}$, we define $C(\mathcal{L})=\max _{P \in \mathcal{L}} C(P)$, and $c(\mathcal{L})=$ $\min _{P \in \mathcal{L}} c(P)$.

We have the following lemma [1] (section 2.6, page 1020).

Lemma 9. Given a polynomial $P$ with coefficients in an ordered domain $\mathbf{D}$, contained in a real closed field $\mathbf{R}$, the greatest absolute value of the roots of $P$ is smaller than $C(P)$, while the smallest absolute value of the non-zero roots of $Q$ is greater than $c(P)$.

Replace $\Omega$ in the polynomial $Q_{\Omega}$ by $A=C(\mathcal{L})$ to obtain a polynomial $Q_{A}$. Replace $\Omega$ in the output roadmap by $A$ to obtain a roadmap $R\left(Z\left(Q_{A}\right), \mathcal{M}\right)$. When projected on $\mathbf{R}^{k}$, this roadmap gives a roadmap $R(Z(Q), \mathcal{M}) \cap B(0, A)$. We next collect all the points $\left(y_{1}, \ldots, y_{k}\right)$ in the roadmap which satisfy $y_{1}^{2}+\ldots+y_{k}^{2}=A$. Each such point is described by a univariate representation involving $\Omega$. We add the curve obtained by treating $\Omega$ as a parameter and letting $\Omega$ vary over $[A, \infty]$, to get a roadmap $R(Z(Q), \mathcal{M})$.

The correctness of the algorithm in the unbounded case follows easily from the correctness of the algorithm in the bounded case. The complexity of the algorithm in the unbounded case coincides with the complexity of the algorithm in the bounded case.

In the case that $\mathcal{M}$ is empty or a singleton, we have proven the following.

Theorem 1. Let $Q \in \mathbf{R}\left[X_{1}, \ldots, X_{k}\right]$ be a polynomial whose total degree is at most $d$. There is an algorithm whose output is a roadmap $R(Z(Q))$ of $Z(Q)$. The number of arithmetic operations used by the algorithm in the ring generated by the coeffcients of $Q$ is bounded by $d^{O\left(k^{2}\right)}$. Let $x \in Z(Q)$ be a point which is represented by a real $k$-univariate representation $(u, \sigma)$ of degree $t$. There is an algorithm whose output $C(x, Z(Q))$ is a semi-algebraic path connecting $x$ to $R(Z(Q))$. The number of arithmetic operations used by the algorithm in the ring generated by the coefficients of $Q$ and the coefficients of the polynomials in $u$ is bounded by $\tau^{O(1)} d^{O\left(k^{2}\right)}$.

Proof. The complexity of the roadmap algorithm (with $\mathcal{M}=\emptyset$ ) is clear after the last complexity analysis. The connecting algorithm is as follows: if the first coordinate $x_{1}$ of $x$ is not one of the distinguished values of $R(Z(Q))$ on the $X_{1}$-axis, compute the finite set $\mathcal{M}$ of real $k$-univariate representations whose associated points are the intersections of the hyperplane $X_{1}=x_{1}$ with $R(Z(Q))$ and construct $R\left(Z(Q)_{x_{1}}, \mathcal{M} \cup\{x\}\right)$. Otherwise, go on with the second coordinate. Its complexity is clear after the preceding complexity analysis.

\section{UNIFORM ALGORITHMS}

6.1. Combinatorial level. Given a polynomial $Q$ and a set of polynomials $\mathcal{P}$ we define the combinatorial level of the system $(Q, \mathcal{P})$ to be the minimum number $\ell$ satisfying:

1. No more than $\ell$ of the polynomials in $\mathcal{P}$ have a real zero in common with $Q$.

2. Any real zero common to $Q$ and to $\ell$ polynomials of $\mathcal{P}$ is isolated.

If the combinatorial level of $(Q, \mathcal{P})$ is $\ell$, then for every $P \in \mathcal{P}$ the combinatorial level of $\left(Q^{2}+P^{2}, \mathcal{P} \backslash\{P\}\right)$ is at most $\ell-1$. Note also that the value of the combinatorial level can be found with complexity $s^{\ell+1} d^{O(k)}$ using Theorems 1.3.2 and 4.2.1 of [1].

We say that a finite set of polynomials $\mathcal{P}$ is in general position over $Z(Q)$ of dimension $k^{\prime}$ if no $k^{\prime}+1$ polynomials of this set have a real zero in common with $Q$ 
and if the only real zeroes common to $k^{\prime}$ polynomials of the set and $Q$ are isolated. Thus, if the set of polynomials $\mathcal{P}$ is in general position over $Z(Q)$ of dimension $k^{\prime}$, then the combinatorial level of the system $(Q, \mathcal{P})$ is at most $k^{\prime}$.

6.2. The connecting subroutine. We suppose that the combinatorial level of the system $(Q, \mathcal{P})$ is at most $\ell \leq k$.

Given a sign condition $\sigma \in\{0,1,-1\}^{\mathcal{P}}$ on $\mathcal{P}$, the corresponding weak sign condition $\bar{\sigma}(P)$ is $\geq 0$ if $\sigma(P)=1$, is $\leq 0$ if $\sigma(P)=-1$ and is $=0$ if $\sigma(P)=0$. Denote by $\mathcal{R}(\sigma)=\left\{x \in R^{k} \mid \forall P \in \mathcal{P}, \operatorname{sign}(P(x))=\sigma, Q(x)=0\right\}$ and by $\mathcal{R}(\bar{\sigma})=\left\{x \in R^{k} \mid \forall P \in \mathcal{P}, P(x)=\bar{\sigma}(P), Q(x)=0\right\}$ which are the realizations of $\sigma$ and $\bar{\sigma}$ on $Z(Q)$. For $p \in Z(Q)$ we denote the sign condition on $\mathcal{P}$ at $p$ by $\sigma_{p}$ and say that $\bar{\sigma}_{p}$ is the weak sign condition defined at $p$. We denote by $\mathcal{P}_{p}$ the set of polynomials of $\mathcal{P}$ vanishing at $p$.

The connecting subroutine takes as input a point $p \in Z(Q)$, described by polynomials of degree at most $\tau$. The output is a semi-algebraic path $\Gamma_{p} \subset \mathcal{R}\left(\bar{\sigma}_{p}\right)_{\pi(p)}$ which connects $p$ to some roadmap $R\left(Z\left(Q, \mathcal{P}^{\prime}\right)\right)$ where $\mathcal{P}^{\prime}$ is a subset of $\mathcal{P}$. The path $\Gamma_{p}$ consists of a sequence of semi-algebraic paths $\gamma_{p, 1}$ joining $p=p_{0}$ to $p_{1}, \gamma_{p, 2}$ joining $p_{1}$ to $p_{2}$, up to $\gamma_{p, j}$ joining $p_{j-1}$ to $p_{j}$ with $j \leq \ell$. Each $\gamma_{p, i}$ is contained in $Z\left(Q, \mathcal{P}_{p_{i-1}}\right)$, and no polynomial of $\mathcal{P} \backslash \mathcal{P}_{p_{i-1}}$ vanishes on $\gamma_{p, i}$. These properties of $\Gamma_{p}$ make clear that $\Gamma_{p}$ is contained in $\mathcal{R}\left(\bar{\sigma}_{p}\right)$.

The algorithm proceeds as follows: First construct a roadmap $R\left(Z\left(Q, \mathcal{P}_{p}\right)\right)$. Compute the set $\mathcal{M}_{p, \mathcal{P}}$ which consists of the real $k$-univariate representations representing the points in $R\left(Z\left(Q, \mathcal{P}_{p}\right)\right)_{\pi(p)}$. Next, construct the roadmap

$$
R\left(Z\left(Q, \mathcal{P}_{p}\right)_{\pi(p)},\{p\} \cup \mathcal{M}_{p, \mathcal{P}}\right) .
$$

If $p$ is connected to $R\left(Z\left(Q, \mathcal{P}_{p}\right)\right)$ by a path $\Gamma$ where no polynomial of $\mathcal{P} \backslash \mathcal{P}_{p}$ vanishes, the connection is done and the algorithm terminates. Otherwise, suppose that $\Gamma \subset R\left(Z\left(\mathcal{P}_{p}\right)_{\pi(p)},\{p\}\right)$ connects $p$ to $R\left(Z\left(\mathcal{P}_{p}\right)\right)$ and a polynomial of $\mathcal{P} \backslash \mathcal{P}_{p}$ vanishes on $\Gamma$. Given $p^{\prime} \in \Gamma$, denote by $\Gamma_{p^{\prime}}$ the connected subset of $\Gamma$ connecting $p$ to $p^{\prime}$. Let $p_{1}$ be the point of $\Gamma$ such that

- $\mathcal{P}_{p_{1}}$ strictly contains $\mathcal{P}_{p}$,

- no $\mathcal{P} \backslash \mathcal{P}_{p}$ vanishes on $\Gamma_{p_{1}} \backslash\left\{p_{1}\right\}$.

Replace $p$ by $p_{1}$ and iterate. Since the combinatorial level of $(Q, \mathcal{P})$ is bounded by $\ell$, the algorithm terminates after $\ell^{\prime} \leq \ell$ iterations. The degrees of the univariate representations representing $p_{1}, \ldots, p_{\ell^{\prime}}$ are bounded by $\tau d^{O\left(k^{2}\right)}$. Thus the complexity of the connecting subroutine is clearly $\ell s \tau^{O(1)} d^{O\left(k^{2}\right)}$. The number of different curve segments in the connecting path is at most $\ell d^{O\left(k^{2}\right)}$.

6.3. Uniform roadmap. In subsection 6.5 we describe an algorithm with input,

1. a polynomial $Q$ for which $Z(Q)$ is bounded,

2. a set of at most $s$ polynomials $\mathcal{P}$ such that the combinatorial level of the system $(Q, \mathcal{P})$ is at most $\ell$.

We further assume that $d$ is a bound on the degree of $Q$ as well as on all the polynomials in $\mathcal{P}$. The algorithm outputs a one-dimensional semi-algebraic set $R(Q, \mathcal{P})$ which is contained in $Z(Q)$ and is called a uniform roadmap of $(Q, \mathcal{P})$. A uniform roadmap of $(Q, \mathcal{P})$ is a union of open curve segments and points satisfying 
the following two conditions:

URM1 The signs of the polynomials $P \in \mathcal{P}$ are constant on each curve segment.

URM2 The intersection of this set with any basic closed semi-algebraic set $S_{\sigma}$, defined by $Q=0$ and a weak sign condition $\sigma$ on $\mathcal{P}$, is a roadmap $R(\mathcal{R}(\sigma))$.

The basic idea behind the algorithm is simple. First, for each at most $\ell$ element subset $\mathcal{P}^{\prime}$ of $\mathcal{P}$ we construct a roadmap for the algebraic set defined by both $Q$ and $\mathcal{P}^{\prime}$, i.e. $Z\left(\{Q\} \cup \mathcal{P}^{\prime}\right)$. We next recurse at each pair $\left(v, \mathcal{P}^{\prime}\right)$, where $v$ is a distinguished value and $Z\left(\mathcal{P}^{\prime}\right)$ is its associated algebraic set. In the recursion, $Q$ is replaced by the algebraic set $Z\left(\{Q\} \cup \mathcal{P}^{\prime}\right)$.

6.4. Finger Subroutine. We describe a subroutine which, given a polynomial $Q$, a set of polynomials $\mathcal{P}$ and a point $p$ in $Z(Q)$ with $\pi(p)=v$, constructs a finite number of semi-algebraic paths starting at $p$ so that every semi-algebraically connected component of every realizable sign condition of $\mathcal{P}$ in $Z(Q)$ sufficiently near and to the left of $p$ contains one of these paths without the point $p$. The subroutine takes as input a polynomial $Q$ and a finite set of polynomials $\mathcal{P}$ for which the combinatorial level of the system $(Q, \mathcal{P})$ is bounded by $\ell$ and a point $p \in Z(Q)$ defined by polynomials of degree at most $d^{O(k)}$. We consider the set $\Sigma_{p}$ of sign conditions $\sigma$ such that $\mathcal{R}(\sigma) \cap Z(Q) \cap B(p, r)_{<v}$ is non-empty for some sufficiently small $r$. The output is a set of semi-algebraic paths starting at $p$ with the property that for some sufficiently small $r$ and for every $\sigma$ in $\Sigma_{p}$ every semialgebraically connected component of $\mathcal{R}(\sigma) \cap Z(Q) \cap B(p, r)_{<v}$ contains one of these paths without the end point $p$. Let the set of polynomials in $\mathcal{P}$ (possibly empty) that are zero at $p$ be $\mathcal{P}_{p}$, and let $B(p, \epsilon)$ be a ball of radius $\epsilon$ and center $p$, where $\epsilon$ is an infinitesimal. Using the Sample Points on a Variety Subroutine with the polynomials defining $B(p, \epsilon)_{<v}$ along with the polynomials $\mathcal{P}_{p}$ as input, we find a point $p_{i}(\epsilon)$ in every semi-algebraically connected component of every non-empty sign condition of the polynomials in $\mathcal{P}_{p}$ in $B(p, \epsilon)_{<v}$. Moreover, $\lim _{\epsilon}\left(p_{i}\right)=p$. For a small enough $t_{0}$, replacing $\epsilon$ by $t$ where $0 \leq t \leq t_{0}$ gives for each $p_{i}(\epsilon)$ semialgebraic paths $p_{i}(t)$, which join $p$ to points in every semi-algebraically connected component of the non-empty sign conditions of $\mathcal{P}$ intersected with $Z(Q) \cap B(p, \epsilon)_{<v}$. The complexity of this subroutine is easily seen to be the cost of calling the Sample Points Subroutine, with $O(\ell)$ polynomials, in $k$ variables, $X_{1}, \ldots, X_{k}$. Since at most $\ell$ polynomials can be zero at $p$, we have at most $3^{\ell}$ conditions to consider at $p$. Using the proof of the complexity bound of the Sample Points Subroutine in [1], we conclude that the complexity of the Finger Subroutine is $3^{\ell} d^{O(k)}$.

6.5. The Uniform Roadmap Algorithm. Note that if the combinatorial level of the system $(Q, \mathcal{P})$ is 0 , then the roadmap $R(Z(Q))$ is a uniform roadmap for $(Q, \mathcal{P})$ since on every semi-algebraically connected component of $Z(Q)$ the signs of the polynomials in $\mathcal{P}$ are fixed and not zero. Moreover, this algorithm has complexity $s d^{O\left(k^{2}\right)}$. If $k=1$, then we can sort the roots of the univariate polynomial $Q$. The roadmap consists of the zeroes of $Q$ together with the signs of the polynomials in $\mathcal{P}$ at these points. The algorithm has complexity $s d^{O(1)}$. The algorithm will call itself recursively and in each recursive call either the combinatorial level or the number of variables will strictly decrease. The two base cases, when the combinatorial level is zero or when the number of variables is one, have been discussed above. 
Input A polynomial $Q$ of degree at most $d$ for which $Z(Q)$ is bounded, a set of polynomials $\mathcal{P}$ whose degrees are bounded by $d$ for which the combinatorial level of the system $(Q, \mathcal{P})$ is bounded by $\ell$.

Output A semi-algebraic set $R(Q, \mathcal{P})$ satisfying conditions URM1 and URM2 of section 6.3 .

Step 0 If $\ell=0$ or if $k=1$, compute $R(Q, \mathcal{P})$ and stop.

Step 1 Use the Curve Segment Subroutine for the sets $Z(Q)$ and $Z\left(Q, \mathcal{P}^{\prime}\right)=$ $Z\left(Q^{2}+\sum_{p \in \mathcal{P}^{\prime}} P^{2}\right)$ for each $\mathcal{P}^{\prime} \subset \mathcal{P}$ of cardinality at most $\ell$. We label each curve segment constructed on $Z\left(Q, \mathcal{P}^{\prime}\right)$ by that algebraic set. The end points of these curve segments are also labeled by the algebraic set $Z\left(Q, \mathcal{P}^{\prime}\right)$ and are placed in a set of distinguished points. Their first coordinates are placed in a set of distinguished values.

Step 2 Compute the intersection of each of these curve segments, with $Z(P)$ for each $P \in \mathcal{P}$. Note that the intersection of a curve segment with the zero set of a polynomial is either the segment itself, or a finite set of points (possibly empty), and we check this by substituting the univariate representation of the curve segment into each polynomial in $\mathcal{P}$ and checking whether the resulting univariate polynomial vanishes identically or not. If the intersection is the curve segment itself, we ignore this intersection. Otherwise, the points of intersection yield a partition of the curve segment. We add these points to our set of distinguished points. If $Z\left(Q, \mathcal{P}^{\prime}\right)$ is the algebraic set labeling a given curve segment, and $P$ is a polynomial which vanishes at one of these new end points, then $Z\left(Q, \mathcal{P}^{\prime} \cup\{P\}\right)$ is the algebraic set that labels this new end point. The signs of the polynomials of $\mathcal{P}$ do not change on any segment of this partition. Moreover, we store the sign vector of the set of polynomials $\mathcal{P}$ on each curve segment and point computed above. The $X_{1}$ coordinates of all distinguished points are computed and appended to the set of distinguished values.

Step 3 For every distinguished point $p$ and algebraic set $Z\left(Q, \mathcal{P}^{\prime}\right)$ which labels $p$ compute the set $\mathcal{M}_{p, \mathcal{P}^{\prime}}$ which consists of the real $k$-univariate representations representing the points of intersection of the curves constructed on $Z\left(Q, \mathcal{P}^{\prime}\right)$ in Step 1 , and the slice $\pi^{-1}(v)$ where $v=\pi(p)$. Call the algorithm recursively to construct a uniform roadmap, connect the points associated to $\mathcal{M}_{p, \mathcal{P}^{\prime}}$ to $R\left(Q+\sum_{P \in \mathcal{P}^{\prime}} P^{2}, \mathcal{P} \backslash \mathcal{P}^{\prime}\right)$ using the preceding connecting subroutine. Note that the combinatorial level of the system passed to the recursive call is at most $\ell-l$, and the number of variables is $k-1$.

Step 4 For each distinguished point $p$ and the corresponding distinguished slice, use the Finger Subroutine to construct curves joining $p$ to points in every semi-algebraically connected component of every realizable sign condition of the set of polynomials $\mathcal{P}$ intersected with $Z(Q) \cap B\left(p, r_{p}\right)_{<\pi(p)}$, for some small enough $r_{p}$. Let the other end points of these curves be $p_{1}, \ldots, p_{j}$.

Step 5 For each $p_{i}, 1 \leq i \leq j$, in the previous step we compute a roadmap, $\Gamma_{p_{i}}$, for the algebraic set $Z(Q)$ in the slice $X_{1}=\pi\left(p_{i}\right)$, which passes through $p_{i}$ as well as the set of points $\mathcal{M}_{p_{i}}=R(Q)_{\pi\left(p_{i}\right)}$. Compute the intersections of the points and curve segments defining $\Gamma_{p_{i}}$ with $Z(P)$ for each $P \in \mathcal{P}$. Note that $\Gamma_{p_{i}}$ is represented as a set of disjoint curve segments and points each labeled by a real univariate representation over an interval along with a list of adjacencies between the points and curve segments. We construct a graph whose vertices are the points in the above representation and whose 
edges are pairs of vertices which are adjacent to a common curve. We call this graph the graph of $\Gamma_{p_{i}}$. Retain the connected component of the graph of $\Gamma_{p_{i}}$ which contains $p_{i}$ and is contained in $S\left(p_{i}\right)$. We call this set $\Gamma_{p_{i}}^{\prime}$.

Step 6 For each $p_{i}$ in the previous step, either $\Gamma_{p_{i}}^{\prime} \cap \mathcal{M}_{p_{i}} \neq \emptyset$, or $\Gamma_{p_{i}}^{\prime}$ intersects $Z(P)$ at a point $q_{i}$ for some $P \in \mathcal{P}$. In the former case, repeat Step 4 with the next $p_{i}$ in the list. In the latter case, repeat Step 4 with $p_{i}$ replaced by $q_{i}$ and replace the algebraic set $Z(Q)$ by $Z\left(Q^{2}+P^{2}\right)$.

Step 7 We output all the curve segments and distinguished points computed in Steps 2 to 6 , with each labeled by the sign condition it satisfies. This is the set $R(Q, \mathcal{P})$.

6.6. Proof of correctness of the Uniform Roadmap Algorithm. Note that Steps 2, 4, and 6 of the algorithm make it evident that $R(Q, \mathcal{P})$ satisfies condition URM1. We proceed to show that it satisfies condition URM2. Without loss of generality, let $S$ be the semi-algebraic set defined by $Q=0, P \geq 0, \forall P \in \mathcal{P}$ and let $R(S)=S \cap R(Q, \mathcal{P})$.

Proposition 8. The set $R(S)$ is a roadmap of the set $S$.

Proof. We first show that $R(S)$ satisfies RM2. For any $c \in \mathbf{R}$ such that $S_{c}$ is nonempty, and for any semi-algebraically connected component $C$ of $S_{c}$, there exists a semi-algebraically connected component $C^{\prime}$ of a non-empty algebraic set, $Z\left(Q, \mathcal{P}^{\prime}\right)_{c}$, such that $C^{\prime} \subset C$ (see Proposition 2 in [1]). Since in the algorithm we construct curves using the Curves Segment Subroutine on all non-empty algebraic sets of the form $Z\left(Q, \mathcal{P}^{\prime}\right)$, it is clear that $R(S)$ intersects $C$. Thus $R(S)$ satisfies RM2. We next show that $R(S)$ satisfies condition RM1 as well. This is the content of the following two lemmas. Let $v_{1}, \ldots, v_{n}$ be the set of distinguished values computed by the algorithm.

Lemma 10. For $1 \leq i \leq n$, if $R(S)_{\leq v_{i}}$ satisfies condition $R M 1$ for the set $S_{\leq v_{i}}$, then $R(S)_{<v_{i+1}}$ satisfies condition $R M 1$ for the set $S_{<v_{i+1}}$.

Proof. Let $C$ be a semi-algebraically connected component of $S_{<v_{i+1}}$, and let $\Gamma$ be a semi-algebraically connected component of $R(S) \cap C_{\left[v_{i}, v_{i+1}\right)}$. The set $\Gamma_{v_{i}} \neq \emptyset$ since there is no distinguished value in $\left(v_{i}, v_{i+1}\right)$. It is then clear that $R(S) \cap C_{\leq v_{i}} \cup \Gamma$ is semi-algebraically connected. Since $R(S) \cap C_{\leq v_{i}}$ is semi-algebraically connected, the conclusion follows.

Lemma 11. For $1 \leq i \leq n$, if $R(S)_{<v_{i}}$ satisfies condition $R M 1$ for the set $S_{<v_{i}}$, then $R(S)_{\leq v_{i}}$ satisfies condition $R M 1$ for the set $S_{\leq v_{i}}$.

Proof. Let $C$ be a semi-algebraically connected component of $S_{\leq v_{i}}$. We prove that $R(S) \cap C$ is semi-algebraically connected. Let $B_{1}, \ldots, B_{h}$ be the semi-algebraically connected components of $S \cap C_{<v_{i}}$. Then, by Lemma $5, C=C_{1} \cup \cdots \cup C_{h} \cup C_{h+1} \cup \cdots \cup$ $C_{N}$ where $C_{i}=\bar{B}_{i}$ for $1 \leq i \leq h$ and for $j>h, C_{j}$ is a semi-algebraically connected component of $Z\left(\mathcal{P}_{j}\right) \cap S_{v_{i}}$ where $\mathcal{P}^{\prime} \subset \mathcal{P}$, and $v_{i}$ is a special value of $Z\left(Q, \mathcal{P}^{\prime}\right)$. Let $\Gamma=R(S) \cap C$ and $\Gamma(i)=R(S) \cap C_{i}$ for $1 \leq i \leq N$; then $\Gamma=\bigcup_{i} \Gamma(i)$. First, we claim that each $\Gamma(j)$ is semi-algebraically connected. If $C_{j}$ is a semi-algebraically connected component of $Z\left(Q, \mathcal{P}^{\prime}\right) \cap S_{v_{i}}$, for some $\mathcal{P}^{\prime} \subset \mathcal{P}$, then, since $v_{i}$ is a special value for this algebraic set, $\Gamma(j)$ is semi-algebraically connected by Step 3 of the algorithm. Otherwise, by the hypothesis of the lemma, we know that $\Gamma(j)_{<v_{i}}$ is semi-algebraically connected. Thus, $\Gamma(j)$ can have at most one semi-algebraically 
connected component whose intersection with $\pi^{-1}\left(-\infty, v_{i}\right)$ is non-empty, and all the other semi-algebraically connected components of $\Gamma(j)$ must lie in $\pi^{-1}\left(v_{i}\right)$, and hence each of these must contain a distinguished point. But, by Steps 4,5 and 6 of our algorithm, the distinguished points get connected to $\Gamma(j)_{<v_{i}}$. Thus, $\Gamma(j)$ can have only one semi-algebraically connected component. Moreover, if $C_{j} \cap C_{j}^{\prime} \neq \emptyset$, then $\Gamma(j)$ and $\Gamma\left(j^{\prime}\right)$ are connected in $R(S)$. This is so since according to Lemma 6 , $C_{j} \cap C_{j}^{\prime}$ intersects an algebraic set which has $v_{i}$ as a special value, and thus contains a distinguished point which gets linked to both $\Gamma(j)$ and $\Gamma\left(j^{\prime}\right)$. It follows easily that $\Gamma$ is semi-algebraically connected. This proves the lemma.

The proposition now follows by induction on $i$.

6.7. Complexity. When $\ell=0$, it follows from the analysis of the algebraic case that the complexity is $s d^{O\left(k^{2}\right)}$. When $k=1$, the complexity is $s d^{O(1)}$. In Step 1, the total number of calls to the Curve Segments Subroutine is $\sum_{1 \leq j<\ell}\left(\begin{array}{c}s \\ j\end{array}\right)$, and each call $\operatorname{costs} d^{O(k)}$. Thus, the total cost of the calls to the Curve Segments Subroutine is bounded by $s^{\ell-1} d^{O(k)}$ arithmetic operations in $\mathbf{D}$. In Step 2, the cost of computing the intersection of the curves computed with the zero sets of the polynomials in $\mathcal{P}$ is bounded by $s^{\ell} d^{O(k)}$, and the total cost of Step 2 is $s^{\ell} d^{O(k)}$. In Step 4 the total cost of the calls to the Finger Subroutine and linking is bounded by $3^{\ell} s^{\ell+1} d^{O(k)}$. We next count the recursive calls. For each $j, 0 \leq j<\ell$, we make $\left(\begin{array}{c}O(s) \\ j\end{array}\right) d^{O(k)}$ recursive calls to the algorithm with the system having combinatorial level $\ell-j$ and geometric dimension $k-1$. Let $T(s, d, \ell, k)$ denote the number of arithmetic operations needed for the problem with these parameters. Since at any depth of the recursion the cost of a single arithmetic operation is bounded by $d^{O\left(k^{2}\right)}$ arithmetic operations in $\mathbf{D}$, we ignore the fact that the ring changes as we go down in the recursion. Thus, we have the following recurrence:

$$
\begin{gathered}
T(s, d, \ell, k) \leq \sum_{0 \leq j \leq \ell}\left(\begin{array}{c}
O(s) \\
j
\end{array}\right) d^{O(k)} T(s, d, \ell-j, k-1)+O\left(3^{\ell} s^{\ell+1} d^{O(k)}\right), \ell>0, k>1, \\
T(s, d, 0, k)=s d^{O\left(k^{2}\right)}, k>1, \\
T(s, d, \ell, 1)=s d^{O(1)} .
\end{gathered}
$$

This recurrence solves to $T(s, d, \ell, k)=3^{\ell} s^{\ell+1} d^{O\left(k^{2}\right)}$. It follows immediately that the total cost is still bounded by $3^{\ell} s^{\ell+1} d^{O\left(k^{2}\right)}$. If $x \in Z(Q)$ is a point represented by a real $k$-univariate representation $(u, \sigma)$ of degree $\tau$, the complexity of connecting $x$ to some $R\left(Q, \mathcal{P}^{\prime}\right)$ is $\ell s \tau^{O(1)} d^{O\left(k^{2}\right)}$ by the complexity of the connecting subroutine.

Thus we have proven the following theorem.

Theorem 2. Given $Q \in \mathbf{R}\left[X_{1}, \ldots, X_{k}\right]$ with $Z(Q)$ bounded, a set of at most $s$ polynomials $\mathcal{P} \subset \mathbf{R}\left[X_{1}, \ldots, X_{k}\right]$ such that the combinatorial level of the system $(Q, \mathcal{P})$ is at most $\ell$, and for which the degrees of the polynomials in $\mathcal{P}$ and $Q$ are bounded by $d$. There is an algorithm which computes a uniform roadmap $R(Q, \mathcal{P})$ using $3^{\ell} s^{\ell+1} d^{O\left(k^{2}\right)}$ arithmetic operations in the ring generated by the coefficients of $Q$ and the coefficients of the elements of $\mathcal{P}$. Given $p \in Z(Q)$ represented by a real $k$-univariate representation $(u, \sigma)$ of degree $\tau$ there is an algorithm which constructs a semi-algebraic path from $p$ to $R(Q, \mathcal{P})$. This algorithm uses $\ell s \tau^{O(1)} d^{O\left(k^{2}\right)}$ 
arithmetic operations in the ring generated by the coefficients of $Q$, the coefficients of the elements of $\mathcal{P}$ as well as the coefficients of the polynomials in the real $k$ univariate representation $(u, \sigma)$.

\section{RoAdMAP ALGORIthm FOR THE BOUNDED CASE}

7.1. Basic idea. We give a roadmap algorithm for an arbitrary semi-algebraic set $S$, defined by the finite set of polynomials $\mathcal{P}$ which is contained in a bounded algebraic variety $Z(Q)$ of real dimension $k^{\prime}$. We remove the boundedness restriction in section 8 . The idea is to construct uniform roadmaps for a perturbed finite set of polynomials which are in general position over approximating varieties which are close to $Z(Q)$ and of dimension $k^{\prime}$. Thus the combinatorial level is $k^{\prime}$. We then take the limits of the curves obtained when the parameter of deformation tends to 0, i.e. the images of the curves so constructed under a lim map, using a subroutine described at the end of this section. In order to ensure that the union of the curves so constructed is semi-algebraically connected in every semi-algebraically connected component, we need to add curves connecting well chosen points to the various roadmaps on approximating varieties. These points are of three kinds:

1. linking points which ensure that roadmaps coming from different approximating varieties whose images under the lim map intersect get connected,

2. touching triples ensuring that if the union of two semi-algebraically connected components of two different sign conditions is semi-algebraically connected, the union of the curves constructed in the two semi-algebraically connected components is semi-algebraically connected and

3. added points that we add on every approximating variety to ensure that the linking points and the touching triples will be part of the final roadmap.

The approximating varieties are constructed as follows in [22] (see also [3]). Let $Z(Q)$ be a real algebraic variety of dimension $k^{\prime}$. Suppose that $Z(Q) \subset B(0, M)$. We assume that $Q$ has degree at most $d$ and is non-negative. This assumption causes no loss of generality as we can replace $Q$ with $Q^{2}$ at the cost of doubling the degree of $Q$. For any index set $I=\left\{i_{k^{\prime}+1}, \ldots, i_{k}\right\} \subset[1, k]$ and an infinitesimal $\delta$, let

$$
\begin{aligned}
& a=\left(k-k^{\prime}\right) M^{-2(d+1)}, \\
& Q_{I}=(1-\delta) Q-\delta\left(X_{i_{k^{\prime}+1}}^{2(d+1)}+\cdots+X_{i_{k}}^{2(d+1)}-a\right), \\
& Q_{I, T}=(1-T) Q-T\left(X_{i_{k^{\prime}+1}}^{2(d+1)}+\cdots+X_{i_{k}}^{2(d+1)}-a\right) \text {, } \\
& \mathcal{Q}_{I}=\left\{Q_{I}, \frac{\partial Q_{I}}{\partial X_{i_{k^{\prime}+2}}}, \ldots, \frac{\partial Q_{I}}{\partial X_{i_{k}}}\right\}, \\
& V_{I}=Z\left(\mathcal{Q}_{I}\right), \\
& W=\bigcup_{I \subset[1, k], \operatorname{card}(I)=k-k^{\prime}} V_{I} .
\end{aligned}
$$

With these notations, the following key result appears in [22].

Proposition 9. The real dimension of $V_{I}$ is at most $k^{\prime}$. For every $x \in Z(Q)$ there exists $y \in W$ such that $\lim _{\delta}(y)=x$. 
7.2. Linking points. Now we describe the construction of the set $L$ of linking points. Note that, by Proposition 4

$$
\lim _{\delta}\left(V_{I}\right)=\operatorname{closure}\left(\left\{(X, T) \in \mathbf{R}^{k} \times \mathbf{R}_{>0} \mid Q_{I, T}(X)=0\right\}\right) \cap\{T=0\} .
$$

If $S$ is a semi-algebraic subset of $\mathbf{R}^{k}$ described by a quantifier-free formula, $\phi(X)$, then the closure of $S$ is described by the following quantified formula:

$$
\psi(X):=\forall Z \exists Y\|X-Y\|^{2}<Z^{2} \wedge \phi(Y) .
$$

Note that $\psi(X)$ is a first-order formula with two blocks of quantifiers, the first with one variable and the second with $k$ variables. Denote by $\mathcal{R}_{I}$ the set of polynomials in $k+1$ variables obtained after two steps of the Block Elimination Subroutine in [1] applied to the polynomials appearing in the first order formula describing closure $\left(\left\{(X, T) \in \mathbf{R}^{k} \times \mathbf{R}_{>0} \mid Q_{I, t}(x)=0\right\}\right)$ in order to eliminate $Z$ and $Y$. These polynomials have the property that closure $\left(\left\{(X, T) \in \mathbf{R}^{k} \times \mathbf{R}_{>0} \mid Q_{I, T}(X)=0\right\}\right)$ is the union of semi-algebraically connected components of sets defined by sign conditions over $\mathcal{R}_{I}$ (note that we do not say that closure $\left(\left\{(X, T) \in \mathbf{R}^{k} \times \mathbf{R}_{>0} \mid\right.\right.$ $\left.Q_{I, T}(X)=0\right\}$ ) can be described by polynomials in $\left.\mathcal{R}_{I}\right)$. According to section 5.2 of [1] the set $\mathcal{R}_{I}$ has $d^{O(k)}$ polynomials and each of these polynomials has degree at most $d^{O(k)}$. Denote by $\mathcal{P}_{I}$ the set of polynomials in $k$ variables obtained by substituting 0 for $T$ in $\mathcal{R}_{I}$. Next, for each pair of multi-indices $I, J$ we apply the Sample Point on a Variety Subroutine, with input $Z(Q), \mathcal{P} \cup \mathcal{P}_{I} \cup \mathcal{P}_{J}$ to obtain the set $L_{I, J}$. The set $L_{I, J}$ consists of $s^{k^{\prime}} d^{O\left(k^{2}\right)}$ points defined by polynomials of degree at most $d^{O\left(k^{2}\right)}$. The complexity of the algorithm just described is $s^{k^{\prime}+1} d^{O\left(k^{2}\right)}$. The set $L$ is the union of the $L_{I, J}$. Thus, for every $I$ and $J, L$ meets every semialgebraically connected component of every non-empty weak sign condition of $\mathcal{P}$ on $\lim _{\delta}\left(V_{I}\right) \cap \lim _{\delta}\left(V_{J}\right)$.

7.3. Touching triples. The reason for constructing the touching triples is the following. Consider two connected components $C_{1}$ and $C_{2}$ of two distinct sign conditions of the family $\mathcal{P}$, which are contained in the set $S$. Though $C_{1}$ and $C_{2}$ are clearly disjoint, $C_{1} \cup C_{2}$ might be connected. If $C_{1} \cup C_{2}$ is connected, then we want the intersection of the roadmap, $R(S)$, with $C_{1} \cup C_{2}$ to be connected as well. We will use the touching triples in the construction of $R(S)$ to insure this property. We compute a set $N$ of touching triples $\left(p_{1}, p_{2}, \gamma_{1,2}\right)$, where $p_{1}, p_{2} \in Z(Q)$, and $\gamma_{1,2}$ is a semi-algebraic path joining $p_{1}$ to $p_{2}$ in $Z(Q)$. The set $N$ has the property that for any two semi-algebraically connected components, $C_{1}$ and $C_{2}$, of sets $\mathcal{R}\left(\sigma_{1}\right)$ and $\mathcal{R}\left(\sigma_{2}\right)$, where $\sigma_{1}, \sigma_{2}$ are strict sign conditions on $\mathcal{P}$, for which $\bar{C}_{1} \cap C_{2} \neq \emptyset$, there exists $\left(p_{1}, p_{2}, \gamma_{1,2}\right) \in N$, such that $p_{1} \in C_{1}, p_{2} \in C_{2}$ and $\gamma_{1,2} \backslash\left\{p_{2}\right\} \in C_{2}$. Thus, if $C_{1}$ and $C_{2}$ are two semi-algebraically connected components of two distinct sign conditions which are included in $S$ and whose union is semi-algebraically connected, then there exists $\left(p_{1}, p_{2}, \gamma_{1,2}\right) \in N$ such that $\gamma_{1,2}$ connects the point $p_{1} \in C_{1}$ with the point $p_{2} \in C_{2}$ by a semi-algebraic path lying in $C_{1} \cup C_{2}$. To compute the set $N$ we introduce two infinitesimals $\eta^{\prime} \ll \eta$ (where $\eta^{\prime} \ll \eta$ means that $\eta^{\prime}$ is infinitesimal over $\mathbf{R}\langle\eta\rangle)$ and let $\mathcal{P}^{\prime}=\bigcup_{P \in \mathcal{P}}\left\{P, P \pm \eta^{\prime}, P \pm \eta\right\}$. We call the Sample Points on a Variety Subroutine with input $Z(Q), \mathcal{P}^{\prime}$ to obtain a set of points in $\mathbf{R}\left\langle\eta, \eta^{\prime}\right\rangle^{k}$ which intersects every semi-algebraically connected component of a set defined by a sign condition on $\mathcal{P}^{\prime}$. For each point $p_{1}$ constructed above, we construct the point $p_{2}=\lim _{\eta^{\prime}}\left(p_{1}\right)$. The point $p_{1}$ is represented as a real $k$-univariate representation $(u, \sigma)$ with coefficients in $\mathbf{D}\left[\eta, \eta^{\prime}\right]$. Replacing $\eta^{\prime}$ in $u$ by a new variable $t$, and letting 
$t$ vary over the interval $\left[0, \eta^{\prime}\right]$ we get a semi-algebraic path $\gamma_{1,2}$ joining $p_{1}$ to $p_{2}$. We include the triple $\left(p_{1}, p_{2}, \gamma_{1,2}\right)$ in the set $N$. We now show that the triples computed above satisfy the property stated at the beginning of this subsection.

Lemma 12. Let $\mathcal{P}=\left\{P_{1}, \ldots, P_{s}\right\}$ be a finite set of polynomials, and suppose that $C_{1}$ and $C_{2}$ are two semi-algebraically connected components of $\mathcal{R}\left(\sigma_{1}\right)$ and $\mathcal{R}\left(\sigma_{2}\right)$ respectively, with $\sigma_{1}, \sigma_{2}$ two strict sign conditions, $\sigma_{1} \neq \sigma_{2}$ and $\bar{C}_{1} \cap C_{2} \neq \emptyset$. Reordering the polynomials in $\mathcal{P}$ if needed there exists $\ell$ and $m$ with $\ell<m$ such that the sign condition $\sigma_{1}$ is $P_{i}=0,1 \leq i \leq \ell$, and $P_{i}>0, m<i \leq s$, while $\sigma_{2}$ is $P_{i}=0,1 \leq i \leq m$, and $P_{i}>0, m<i \leq s$. Taking infinitesimals, $\eta^{\prime} \ll \eta$, there is a semi-algebraically connected component $C$, of the set defined by the sign condition $P_{i}=0,1 \leq i \leq \ell, 0<P_{i}<\eta^{\prime}, \ell<i \leq m, P_{i}>\eta, m<i \leq s$, such that $C \subset C_{1 \mathbf{R}\left\langle\eta, \eta^{\prime}\right\rangle}$, and $\lim _{\eta^{\prime}}(C) \subset C_{2 \mathbf{R}\left\langle\eta, \eta^{\prime}\right\rangle}$.

Proof. Choose a point $p \in \bar{C}_{1} \cap C_{2}$. Since $p \in C_{2}, \mathcal{P}$ must satisfy the sign condition $\sigma_{2}$ at $p$. Since $p \in \bar{C}_{1}$, any infinitesimal neighborhood (infinitesimal with respect to $\left.\mathbf{R}\left\langle\eta, \eta^{\prime}\right\rangle\right)$ of $p$ must intersect $C_{1}$. Consider the ball $B(p, \epsilon)$ centered at $p$, with radius $\epsilon$, where $\epsilon$ is any infinitesimal over $\mathbf{R}\left\langle\eta, \eta^{\prime}\right\rangle$. Then, there exists a point $q \in B(p, \epsilon) \cap$ $C_{1}$ and hence $\mathcal{P}$ must satisfy the sign condition $\sigma_{1}$ at $q$. That is, $P_{i}=0,1 \leq i \leq \ell$, and $P_{i}>0, m<i \leq s$, at $q$. $\operatorname{Since} \lim _{\epsilon}(q)=p$, if a polynomial is zero at $q$ it must also be zero at $p$, and if a polynomial is positive at $q$, it must be non-negative at $p$. This proves that $\sigma_{2}$ has the form announced. It is clear that $q \in\left(\mathbf{R}\left\langle\eta, \eta^{\prime}, \epsilon\right\rangle\right)^{k}$ satisfies the inequalities $P_{i}=0,1 \leq i \leq \ell, 0<P_{i}<\eta^{\prime}, \ell<i \leq m, P_{i}>\eta, m<$ $i \leq s, P_{1}=\cdots=P_{\ell}=0,0<P_{\ell+1}<\eta^{\prime}, \ldots, 0<P_{m}<\eta^{\prime}, P_{m+1}>\eta, \ldots, P_{s}>\eta$. Consider the semi-algebraically connected component $C$ of the set defined by these inequalities that contains $q$. It is clear that $C \subset C_{1}$. Moreover, $\lim _{\eta^{\prime}}(C)$ satisfies the sign condition $\sigma_{2}$ and contains $p \in C_{2}$. Since $\lim _{\eta^{\prime}}$ maps semi-algebraically connected sets to semi-algebraically connected sets, we see that $\lim _{\eta^{\prime}}(C) \subset C_{2}$.

It is clear that if $p$ is a point in $C$, if $q=\lim _{\eta^{\prime}}(p)$, and if $\gamma$ is the path obtained by replacing $\eta^{\prime}$ by a variable as in the algorithm described earlier, then $p \in C_{1}$, $q \in C_{2}$ and $\gamma$ is a path joining $p$ and $q$ contained in $C_{1}$ except at the end point $q$.

7.4. Added points. For every $p \in L \cup N$ and every $I$ such that $V_{I}$ is infinitesimally close to $p$, we construct a point $p_{I}$ in $V_{I}$ infinitesimally close to $p$ as follows. The point $p$ is given by a real univariate representation, $u=\left(f, g_{0}, \ldots, g_{k}\right)$ and a Thom encoding $\sigma$ of a real root $t_{\sigma}$ of $f$. Let $\epsilon \gg \delta$ be an infinitesimal, and let $P_{p}\left(X_{1}, \ldots, X_{k}, t_{\sigma}\right)=g_{0}\left(t_{\sigma}\right)^{2} \sum_{i}\left(g_{0}\left(t_{\sigma}\right) X_{i}-g_{i}\left(t_{\sigma}\right)\right)^{2}-g_{0}\left(t_{\sigma}\right)^{2} \epsilon^{2}$. We call the Sample Point on a Variety Subroutine with input $P_{p}, V_{I}$ and perform the computations over the extended ring $\mathbf{D}\left[t_{\sigma}\right]$. At each point obtained we keep all those points at which the sign of $P_{p}$ is negative and discard the rest. We denote by $A_{I}$ the points of $V_{I}$ so obtained. The set $\mathcal{A}=\bigcup_{I} A_{I}$ is our set of added points. The complexity of computing the set of added points $\mathcal{A}=\bigcup_{I} A_{I}$ is $s^{k^{\prime}} d^{O\left(k^{2}\right)}$. This follows from the complexity of the Sample Points on a Variety Subroutine used repeatedly during the algorithm.

7.5. The Roadmap Algorithm. We now describe the roadmap algorithm in the general case. Take an infinitesimal $\delta$ and compute the set of added points $\mathcal{A}$. Make a perturbation of the polynomials in $\mathcal{P}$ as follows. Take two more infinitesimals $\epsilon$ and $\epsilon^{\prime}$ with $\delta \ll \epsilon^{\prime} \ll \epsilon$ and replace the set $\mathcal{P}$ by the set $\mathcal{P}^{*}$ which consists of $4 s$ 
polynomials:

$$
\begin{aligned}
\mathcal{P}^{*}= & \bigcup_{i=1, \ldots, s}\left\{\left(1-\epsilon^{\prime}\right) P_{i} \epsilon-\epsilon H_{4 i-3},\left(1-\epsilon^{\prime}\right) P_{i}+\epsilon H_{4 i-2},\right. \\
& \left.\left(1-\epsilon^{\prime}\right) P_{i}-\epsilon^{\prime} \epsilon H_{4 i-1},\left(1-\epsilon^{\prime}\right) P_{i}+\epsilon^{\prime} \epsilon H_{4 i}\right\},
\end{aligned}
$$

where $H_{i}=\left(1+\sum_{1 \leq j \leq k} i^{j} x_{j}^{d^{\prime}}\right)$ and $d^{\prime}$ is an even number greater than the degree of any $P_{i}$. We have the following two lemmas, which are very similar to Propositions 5 and 6 in [3]. They show that the set $\mathcal{P}^{*}$ is in general position over each $V_{I}$, and that there exists a correspondence between the semi-algebraically connected components of realizable sign conditions of $\mathcal{P}$ and $\mathcal{P}^{*}$.

Lemma 13. The combinatorial level of $\left(\mathcal{Q}_{I}, \mathcal{P}^{*}\right)$ is at most $k^{\prime}$.

Lemma 14. Let $\sigma$ be a realizable strict sign condition on the set of polynomials $\mathcal{P}$ on $Z(Q)$ given by $P_{1}=\ldots=P_{\ell}=0, P_{\ell+1}>0, \ldots, P_{s}>0$, and let $C$ be a semi-algebraically connected component of the intersection of the realization $\mathcal{R}(\sigma)$ of $\sigma$ with $Z(Q)$. Let $\sigma^{\prime}$ be the weak sign condition on $\mathcal{P}^{*}$ given by

$$
\begin{aligned}
-\epsilon \epsilon^{\prime} H_{4 i} \leq & \left(1-\epsilon^{\prime}\right) P_{i} \leq \epsilon^{\prime} \epsilon H_{4 i-1}, 1 \leq i \leq \ell, \\
& \left(1-\epsilon^{\prime}\right) P_{i} \geq \epsilon H_{4 i-3},
\end{aligned}
$$

and let $\mathcal{R}\left(\sigma^{\prime}\right)$ be its realization. Then, for every $I$ such that $\lim _{\epsilon^{\prime}}\left(V_{I}\right) \cap \mathcal{R}(\sigma) \neq \emptyset$, there exists a unique semi-algebraically connected component $C^{\prime}$ of $\mathcal{R}\left(\sigma^{\prime}\right) \cap V_{I}$ such that $\lim _{\epsilon^{\prime}}\left(C^{\prime}\right) \subset C_{\mathbf{R}\langle\epsilon\rangle}$. Moreover, if $x \in \mathbf{R}^{k}$ is in $C$, then $x \in C^{\prime}$.

Now use the algorithm for constructing uniform roadmaps with input $\left(\mathcal{Q}_{I}, \mathcal{P}^{*}\right)$. Connect the points of $\mathcal{A}_{I}$ to the uniform roadmap of $\left(\mathcal{Q}_{I}, \mathcal{P}^{*}\right)$ using the connecting subroutine. Then compute the image of the roadmap constructed above under the $\lim _{\epsilon^{\prime}}$ map, using the Limit of a Curve Segment Subroutine and retain only those portions which are in the given set $S$. In order to connect a point $x$ to the roadmap, an $I$ such that $x \in \lim _{\epsilon^{\prime}}\left(V_{I}\right)$ is chosen and a point $x_{I}$ infinitesimally close to $x$ in $V_{I}$ is constructed using the Sample Points Subroutine [1]. This point $x_{I}$ is connected to the uniform roadmap $R\left(\mathcal{Q}_{I}, \mathcal{P}^{*}\right)$ using the connecting subroutine. Then we compute the image of the connecting curves using the Limit of a Curve Segment Subroutine.

The proof of correctness follows from the proof of correctness of the uniform algorithm and Lemmas 13 and 14. The complexity of computing the set of added points $\mathcal{A}$ is $s^{k^{\prime}} d^{O\left(k^{2}\right)}$. The combinatorial level of $\left(\mathcal{Q}_{I}, \mathcal{P}^{*}\right)$ is bounded by $k^{\prime}$. Using the complexity bound of the uniform algorithm, we see that the complexity is bounded by $s^{k^{\prime}+1} d^{O\left(k^{2}\right)}$. Similarly, using the complexity bounds for the connecting subroutine, the complexity of connecting a point $x$ described by polynomials of degree at most $\tau$ to the roadmap is $k^{\prime} s \tau^{O(1)} d^{O\left(k^{2}\right)}$.

\section{Roadmap algorithm in the unbounded Case}

In this section we show how to modify the algorithm presented in section 7 to handle the case when the input variety $Z(Q)$ is not bounded. If $Z(Q)$ is not bounded, we first introduce a new variable $X_{k+1}$ and a new variable $\Omega$ and replace $Q$ by the polynomial $Q_{\Omega}=Q^{2}+\left(X_{1}^{2}+\cdots+X_{k+1}^{2}-\Omega^{2}\right)^{2}$. Let $S_{\Omega} \in R\langle 1 / \Omega\rangle^{k+1}$ be the set defined by the same formula as $S$ but with $Q$ replaced by $Q_{\Omega}$. The roadmap algorithm outputs a road map of $R\left(S_{\Omega}\right)$, composed of points and curves 
whose description involves $\Omega$. Let $\mathcal{L}$ be the set of all polynomials in $\mathbf{D}[\Omega]$ whose signs were determined in the various calls to the Multi-variate Sign Determination Subroutine inside the algorithm. We replace $\Omega$ by $A=C(\mathcal{L})$ (see Definition 2 ) in the output roadmap to obtain a roadmap $R\left(S_{A}\right)$, which, when projected on $\mathbf{R}^{k}$, gives a roadmap $R(S) \cap B(0, A)$. We next collect all the points $\left(y_{1}, \ldots, y_{k}\right)$ in the roadmap which satisfy $y_{1}^{2}+\ldots+y_{k}^{2}=A$. Each such point is described by a univariate representation involving $\Omega$. We add the curve obtained by treating $\Omega$ as a parameter and letting $\Omega$ vary over $[A, \infty]$, to get a roadmap $R(S)$. The complexity of the algorithm in the unbounded case coincides with the complexity of the algorithm in the bounded case. Thus we have proven the following theorem.

Theorem 3. Let $Q \in \mathbf{R}\left[X_{1}, \ldots, X_{k}\right]$ and $\mathcal{P} \subset \mathbf{R}\left[X_{1}, \ldots, X_{k}\right]$ be a set of at most $s$ polynomials each of degree less than $d$. Let $k^{\prime}$ be the real dimension of $Z(Q)$. Given a semi-algebraic $S \subset Z(Q)$ defined by $\mathcal{P}$, there is an algorithm outputting a roadmap $R(S)$ of $S$ which uses $s^{k^{\prime}+1} d^{O\left(k^{2}\right)}$ arithmetic operations in the ring $\mathbf{D}$ generated by the coefficients of $Q$ and the elements of $\mathcal{P}$. Let $x \in Z(Q)$ be a point which is represented by a real $k$-univariate representation $(u, \sigma)$ of degree $t$. There is an algorithm whose output is a semi-algebraic connected path connecting $x$ to $R(S)$. The number of arithmetic operations used by the algorithm in the ring $\mathbf{D}$ generated by the coefficients of $Q$, the coefficients of the elements of $\mathcal{P}$ and the coefficients of the polynomials in $u$ is bounded by $k^{\prime} s \tau^{O(1)} d^{O\left(k^{2}\right)}$.

Corollary 2. Let $Q \in \mathbf{R}\left[X_{1}, \ldots, X_{k}\right]$ and $\mathcal{P} \subset \mathbf{R}\left[X_{1}, \ldots, X_{k}\right]$ be a set of at most s polynomials each of degree less than $d$. Let $k^{\prime}$ be the real dimension of $Z(Q)$. Given a semi-algebraic $S \subset Z(Q)$ defined by $\mathcal{P}$, there is an algorithm outputting exactly one point in each semi-algebraically connected component of $S$ whose complexity is $s^{k^{\prime}+1} d^{O\left(k^{2}\right)}$ arithmetic operations in the ring $\mathbf{D}$ generated by the coefficients of $Q$ and the coefficients of the elements of $\mathcal{P}$.

Note that we do not know how to compute the dimension $k^{\prime}$ of $Z(Q)$ in less than $d^{O\left(k^{2}\right)}$ arithmetic operations [22].

\section{REFERENCES}

[1] S. Basu, R. Pollack, M.-F. Roy On the combinatorial and algebraic complexity of Quantifier Elimination, J. Assoc. Comput. Machin., 43, 1002-1045, (1996). MR 98c:03077

[2] S. Basu, R. Pollack, M.-F. Roy Computing Roadmaps of Semi-algebraic Sets, Proc. 28th Annual ACM Symposium on the Theory of Computing, 168-173, (1996). CMP 97:06

[3] S. Basu, R. Pollack, M.-F. Roy On Computing a Set of Points meeting every Semialgebraically Connected Component of a Family of Polynomials on a Variety, J. Complexity, Vol 13, Number 1, 28-37 (1997). MR 98d:14071

[4] S. Basu, R. Pollack, M.-F. Roy Computing Roadmaps of Semi-algebraic Sets on a Variety, Foundations of Computational Mathematics, F. Cucker and M. Shub, Eds., 1-15, (1997). CMP 99:05

[5] J. Bochnak, M. Coste, M.-F. Roy Real algebraic geometry. Ergebnisse der Mathematik und ihrer Grenzgebiete. 3. Folge, Bd. 36, Berlin: Springer-Verlag (1998). CMP 99:07

[6] J. CAnny, The Complexity of Robot Motion Planning, MIT Press (1987). MR 89m:68142

[7] J. CANnY, Computing road maps in general semi-algebraic sets, The Computer Journal, 36: 504-514, (1993). MR 94i:68108

[8] J. Canny, D. Grigor'ev, N. Vorobjov Finding connected components of a semi-algebraic set in subexponential time, Applic. Alg. Eng. Comput. 2: 217-238 (1992). MR 95m:03078

[9] G. E. Collins, Quantifier elimination for real closed fields by cylindrical algebraic decomposition, Autom. Theor. form. Lang., 2nd GI Conf., Kaiserslautern 1975, Lect. Notes Comput. Sci. 33, 134-183 (1975). MR 53:7771 
[10] M. Coste, M.-F. Roy Thom's lemma, the coding of real algebraic numbers and the topology of semi-algebraic sets, J. Symb. Comput. 5, No.1/2, 121-129 (1988). MR 89g:12002

[11] M. Coste, M. Shiota Nash triviality in families of Nash manifolds, Invent. Math. 108, 349-368 (1992). MR 93e:14066

[12] D. Grigor'ev, N. Vorobjov Counting connected components of a semi-algebraic set in subexponential time, Comput. Complexity 2, No.2, 133-186 (1992). MR 94a:68062

[13] L. Gournay, J. J. Risler Construction of roadmaps of semi-algebraic sets, Appl. Algebra Eng. Commun. Comput. 4, No.4, 239-252 (1993). MR 94j:14049

[14] R. M. HaRdt Semi-algebraic Local Triviality in Semi-algebraic Mappings, Am. J. Math. 102, 291-302 (1980). MR 81d:32012

[15] J. Heintz, M.-F. Roy, P. Solernò Single exponential path finding in semi-algebraic sets I: The case of a regular hypersurface, Discrete and Applied Math., Proc.AAECC-8 Tokyo, 1990; Lectures Notes in Comp. Sci. 508, Springer-Verlag 180-186 (1991). MR 92j:14072

[16] J. Heintz, M.-F. Roy, P. Solernò Single exponential path finding in semi-algebraic sets II : The general case, Bajaj, Chandrajit L. (ed.), Algebraic geometry and its applications. Collections of papers from Shreeram S. Abhyankar's 60th birthday conference held at Purdue University, West Lafayette, IN, USA, June 1-4, 1990. New York: Springer-Verlag, 449-465 (1994). MR 95i:14054

[17] J.-C. Latombe Robot Motion Planning, The Kluwer International Series in Engineering and Computer Science. 124. Dordrecht: Kluwer Academic Publishers Group (1991).

[18] J. Milnor Morse Theory, Princeton: Princeton Univ. Press (1963). MR 29:634

[19] J. Renegar On the computational complexity and geometry of the first order theory of the reals, parts I, II and III., J. Symb. Comput., 13 (3) 255-352, (1992). MR 93h:03011a; MR 93h:03011b; MR 93h:03011c

[20] F. Rouillier, M.-F. Roy, M. SAFey Finding at least a point in each connected component of a real algebraic set defined by a single equation, to appear in Journal of Complexity.

[21] M.-F. Roy, N.N. VorobJov Finding irreducible components of some real transcendental varieties, Journal of Computationnal Complexity 4 107-132 (1994). MR 95g:68056

[22] M.-F. Roy, N. Vorobjov Computing the Complexification of a Semi-algebraic Set, Proc. of International Symposium on Symbolic and Algebraic Computations, 1996, 26-34 (complete version to appear in Math. Zeitschrift).

[23] J. Schwartz, M. Sharir On the 'piano movers' problem II. General techniques for computing topological properties of real algebraic manifolds, Adv. Appl. Math. 4, 298-351 (1983). MR 85h:52014

Department of Mathematics, University of Michigan, Ann Arbor, Michigan 48109

E-mail address: saugata@math.1sa.umich.edu

Courant Institute of Mathematical Sciences, New York University, New York, New YORK 10012

E-mail address: pollack@cims.nyu.edu

IRMaR (URA CNRS 305), Université de Rennes, Campus de Beaulieu 35042 Rennes CEDEX, FRANCE

E-mail address: mfroy@univ-rennes1.fr 